\title{
How to speed up the detection of aerobic microbial contaminations by using isothermal microcalorimetry
}

\author{
Christian Fricke ${ }^{1} \cdot$ Hauke Harms ${ }^{1} \cdot$ Thomas Maskow $^{1}$ id
}

Received: 20 January 2020 / Accepted: 22 June 2020 / Published online: 3 July 2020

(c) The Author(s) 2020

\begin{abstract}
Isothermal microcalorimetry (IMC) is regarded as a promising diagnostic tool for fast detection of bacterial contaminations in various matrices. Based on a reference detection time determined by visual inspection of bacterial growth on solid medium, we investigated the strict aerobically growing Pseudomonas putida mt-2 KT2440 in a static 4-mL ampoule system on solid and liquid media by IMC to evaluate the three main options to reduce the detection time of bacterial contamination. Firstly, the sample preparation (e.g. membrane filtration) leads to an elevated number of bacteria in the measuring ampoule and thus to a reduced detection time. Secondly, the amount of substrate and oxygen has been investigated by varying the filling volume of medium in the calorimetric ampoule. Here, we were able to show how biophysical characteristics like the substrate and oxygen diffusion determined the shape of heat flow signals and thus the detection time. Finally, the technical framework determines the sensitivity of the IMC instrument. We examined the impact of four different detection threshold values $(2,10,50$ and $100 \mu \mathrm{W})$ on the detection time as a function of the initial number of bacteria presented in the ampoule and the filling volume.
\end{abstract}

Keywords Aerobic system $\cdot$ Calorimetric detection $\cdot$ Isothermal microcalorimetry $\cdot$ Pseudomonas putida KT2440 $\cdot$ Realtime monitoring

\section{List of symbols}

$c_{0} \quad$ Initial bacterial concentration of each dilution determined by $\mathrm{CFU}$ counting $\left(\mathrm{CFU} \mathrm{mL} \mathrm{mL}^{-1}\right)$

$c_{\mathrm{O}_{2}} \quad$ Concentration of the dissolved oxygen in the liquid medium $\left(\mathrm{mg} \mathrm{L}^{-1}\right)$

LC Liquid cultivation

LOD Limit of thermal detection (W)

MF Membrane filtration

$N_{0} \quad$ Initial number of bacteria (CFU)

$n_{\text {TEG }} \quad$ Number of thermocouples

SC Solid cultivation

SD Standard deviation

$t \quad$ Time (h)

$t_{\text {dect }} \quad$ Detection time (h)

Electronic supplementary material The online version of this article (https://doi.org/10.1007/s10973-020-09986-0) contains supplementary material, which is available to authorized users.

Thomas Maskow

thomas.maskow@ufz.de

1 Department of Environmental Microbiology, HelmholtzCentre for Environmental Research - UFZ, Leipzig, Germany

$\begin{array}{ll}U & \text { Voltage }(\mathrm{V}) \\ V_{\mathrm{i}} & \text { Inoculum volume }(\mathrm{mL}) \\ V_{\text {medium }} & \text { Filling volume of medium }(\mathrm{mL}) \\ \alpha & \text { Seebeek coefficient }\left(\mathrm{V} \mathrm{K}^{-1}\right) \\ \Delta_{\mathrm{k}} H_{\mathrm{O}_{2}} & \text { Oxycaloric equivalent of }-455 \mathrm{~kJ} \mathrm{~mol}^{-1} \\ \varepsilon & \text { Calibration coefficient }\left(\mathrm{V} \mathrm{W}^{-1}\right) \\ \kappa & \text { Specific thermal conductivity }\left(\mathrm{W} \mathrm{K}^{-1}\right) \\ \mu & \text { Specific growth rate }\left(\mathrm{h}^{-1}\right) \\ \Phi_{\text {dect }} & \text { Detection threshold value }(\mathrm{W}) \\ \Phi & \text { Heat flow }(\mathrm{W}) \\ \varphi_{0} & \text { Cell-specific heat production rate }(\mathrm{W})\end{array}$

\section{Introduction}

In general, a calorimeter is capable of detecting the reaction heat released or taken up by any type of physical, chemical or biological process in real-time [1]. This has led to numerous applications in a wide range of fields, including life sciences [2-6], materials science [7], biotechnology [8, 9] and medical diagnostics [10, 11]. As one of the first applications in biocalorimetry in 1780, Lavoisier and LaPlace linked calorimetry to biology by studying the metabolism of 
guinea pigs [12]. Even the tiny heat released during bacterial growth can be monitored using modern high-performance calorimeters. These micro- or nanocalorimeters operate typically in isothermal mode and allow high resolutions in the nano- to microwatt range [1].

The increase in biomass during bacterial growth proceeds along with the formation of metabolic end products such as water or carbon dioxide. At the molecular level, numerous enzymatically controlled reactions take place that break down energy-rich organic molecules (catabolism) or synthesize biomolecules to maintain metabolism and biomass production (anabolism) [13]. Part of the assimilated Gibbs energy is released into the environment in the form of heat $[2,14]$. One application focus of biocalorimetry is the qualitative and quantitative detection of bacterial contaminations in food [5], drinking water [15] or for sterility testing of pharmaceuticals [16]. Current isothermal microcalorimeters (IMC) do not allow the detection of a single bacterial cell, due to the low heat production rate of bacterial cells (a few $\mathrm{pW}$ ) [17] which overstrains the efficiency of current heat flow sensors [18]. Detecting bacterial contaminations is possible by monitoring the growth of the bacteria in real time by cultivation in liquid (LC) $[15,19-22]$ or solid (SC) [23-25] medium. This benefits from the exponential growth forming substantial biomass from a single bacterial cell within a short time [26]. In this study, we identify the factors influencing the detection time in 4-mL static ampoules in microcalorimetric real-time monitoring using the example of Pseudomonas putida mt-2 KT2440.

Our emphasis is on the interplay between initial bacterial numbers, their substrate provision in $\mathrm{LC}$ and $\mathrm{SC}$, and the performance of the calorimeter. Detection times are compared with those achieved by conventional visual detection (i.e. the counting of colony-forming units; CFUs).

\section{Materials and methods}

\section{Bacterial strain, medium and cultivation}

Pseudomonas putida mt2-KT2440 (German Collection of Microorganisms and Cell Cultures, DSMZ, Braunschweig, Germany) was used for the microcalorimetric experiments. The strain was cultivated on DSM-1 medium, recommended by DSMZ which is composed of (in $\mathrm{g} \mathrm{L}^{-1}$ ): peptone (5.0), meat extract (3.0) and agar (15.0). The liquid pre-cultures of $P$. putida were inoculated by a single colony of a pre-grown Petri dish culture. The liquid pre-culture was incubated overnight at room temperature. The identity of the species was regularly checked by the morphology of the colonies as well as by microscopy before and after the calorimetric experiments.

\section{Calorimetric monitoring}

The microcalorimeter TAM III (TA Instruments, New Castle, USA) with 12-channels as well as the cement calorimeter with 14-channels MC-Cal/100 P (C3 Prozess- und Analysetechnik GmbH, Munich, Germany) and 4.2-mL glass ampoules were used to perform the bacterial growth experiments. The glass ampoules and the caps were autoclaved at $121{ }^{\circ} \mathrm{C}$ for $40 \mathrm{~min}$, subsequently filled with DSM-1 medium or DSM-1 agar and stored in the fridge. The ampoules were warmed up to room temperature before measurements and inoculated with bacteria. In the case of larger filling volumes of medium, the glass ampoules were warmed up in an incubator $\left(30 \pm 0.2^{\circ} \mathrm{C}\right)$ to ensure fast thermal equilibration of the whole sample. The prepared ampoules were then placed into the channels of the microcalorimeter and thermally equilibrated for $15 \mathrm{~min}$ in the pre-heating position. After further $45 \mathrm{~min}$ for thermal equilibration in the measuring position, the heat flow was recorded. Before the experimental set-up was changed (e.g. for modification of filling volume), a gain calibration was performed to ensure the precision of measurements. A single pulse of $1 \mathrm{~mW}$ was generated in each channel by an integrated electrical calibration heater. As a result of the calibration, a gain factor and the offset of the respective channel were calculated.

In the case of the cement calorimeter, an internal electrical calibration was performed before the experiments were conducted, and gain factors, as well as the offset of each channel, were determined. Unlike the microcalorimeter, the prepared ampoules were directly placed in the measuring position. One channel was selected as reference and contained a reference ampoule (filled with $1 \mathrm{~mL}$ sterile medium). All measurements were conducted at $30.0{ }^{\circ} \mathrm{C}$. After the heat flow had returned to the baseline, the measurements were completed and the ampoules were stored in the refrigerator for final microscopic examination.

The final data evaluation was carried out using the Origin 2018 software. Baseline corrections followed the procedure described by [25].

\section{Experimental}

The experiments were divided into two sets: firstly CFU counting on solid culture as reference (Fig. 1) and secondly monitoring bacterial growth on solid culture (SC) (Fig. 2a) as well as in liquid culture (LC) (Fig. 2b) using IMC. In the case of reference experiments, we performed CFU counting experiments to investigate the influence of the initial concentration of bacteria on the detection time (Fig. 1). 
Fig. 1 Experimental set-up for visual inspection (CFU counting) of bacterial growth on solid medium
Visual inspection of CFUs by solid cultivation (SC)

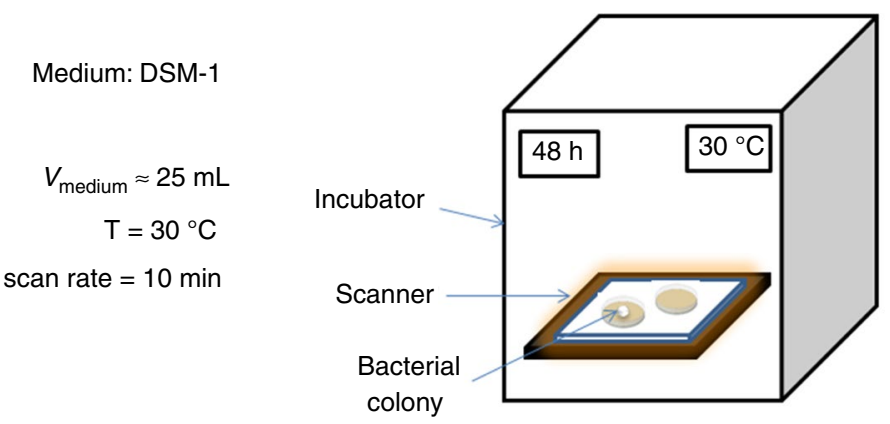

Evalution of obtained images by visual counting of colonies

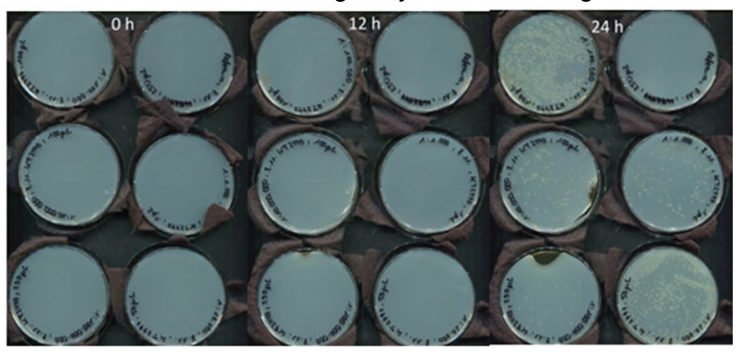

\section{CFU determination and CFU counting}

Starting from a diluted pre-culture with an $\mathrm{OD}_{600}$ of approx. 0.2 a dilution series in 10 -fold steps were prepared. To determine the initial concentration of bacteria $c_{0}$ in $\mathrm{CFU} \mathrm{mL} \mathrm{m}^{-1}$ for each dilution, conventional CFU countings were conducted in parallel. Initial bacterial concentrations were ranging between $10^{5}$ and $10^{1} \mathrm{CFU} \mathrm{mL}^{-1}$.

Common DSM-1 agar plates were inoculated with the abovementioned concentrations (including a sterile blank). As an exception, in one series of experiments, the same initial bacterial concentration $\left(10^{1} \mathrm{CFU} \mathrm{mL} \mathrm{m}^{-1}\right)$ but different inoculum volumes $V_{\mathrm{i}}=0.01,0.1,0.25,0.5$ and $1 \mathrm{~mL}$ were used (result see S1 in Supporting Information, SI). After inoculation, the progress of colony formation was monitored at 10-min intervals using a scanner. The scanner was located in a temperature-controlled incubator (TH 30, Edmund Bühler GmbH, Bodelshause, Germany), at $30 \pm 1{ }^{\circ} \mathrm{C}$ (see Fig. 1). The resulting images were saved as jpg-files and subsequentially evaluated. For this purpose, five independent observers inspected the obtained images. The detection time was defined as the time at which colonies were first discovered. The resulted detection time served as reference values for the IMC experiments and allowed us to compare CFU counting directly with IMC.

\section{Microcalorimetric experiments}

The IMC experiments on solid and in liquid medium (experimental set-up is shown in Fig. 2) were subdivided into three parts: The first part dealt with the initial number of bacteria $N_{0}$ (CFU in the ampoule). Here, the calorimetric ampoules were filled with $1 \mathrm{~mL}$ medium. In the case of $\mathrm{SC}$ and $\mathrm{LC}, 0.01 \mathrm{~mL}$ of each concentration $\left(10^{5}-10^{1} \mathrm{CFU} \mathrm{mL}{ }^{-1}\right.$, including a sterile blank) were added to the medium for inoculation $V_{\mathrm{i}}$ (in $\mathrm{mL}$ ). The initial number of bacteria $N_{0}$ results from the initial concentration $c_{0}$ and the inoculum volume $V_{\mathrm{i}}$. The experiments were performed in triplicates. Further experiments were performed by membrane filtration (SC and LC, $n=1$ ) as well as a range of higher inoculum volumes (LC, $n=1)$. In the former, the influence of the enrichment procedure was investigated and $10 \mathrm{~mL}$ of each concentration $\left(10^{5}-10^{1} \mathrm{CFU} \mathrm{mL} \mathrm{mL}^{-1}\right.$, including a sterile blank) were membrane filtrated and subsequently purged with $2 \mathrm{~mL}$ sterile DSM-1 medium. Therefore, compatible sterile membrane filters (pore size $0.45 \mu \mathrm{m}$, cellulose nitrate, Sartorius AG, Göttingen, Germany) were prepared under sterile conditions using a punch (diameter $10 \mathrm{~mm}$, LocheisenSatz, BGS Technic KG, Wermelskirchen, Germany). The punched membrane filters were placed in specially made sealed adapters which were integrable into a $100-\mathrm{mL}$ 
(a)

Medium: DSM-1

$V_{\text {ampoule }} \approx 4.2 \mathrm{~mL}$

$T=30{ }^{\circ} \mathrm{C}$

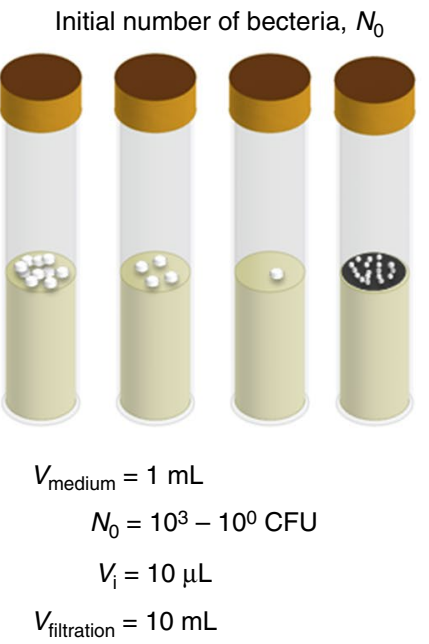

(b)

Medium: DSM-1,

MMKT2440

$$
\begin{array}{r}
V_{\text {ampoule }} \approx 4.2 \mathrm{~mL} \\
T=30^{\circ} \mathrm{C}
\end{array}
$$

Initial number of becteria, $N_{0}$

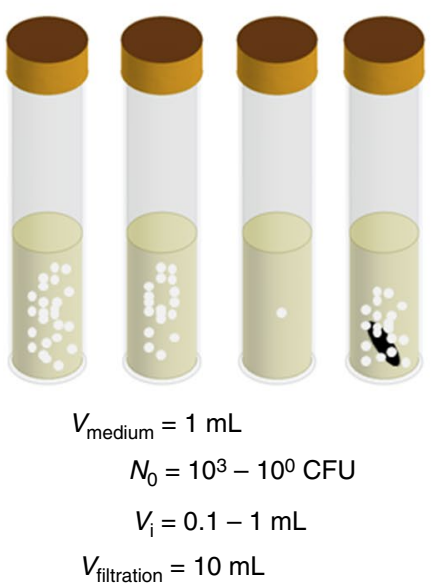

\section{Solid Cultivation (SC)}
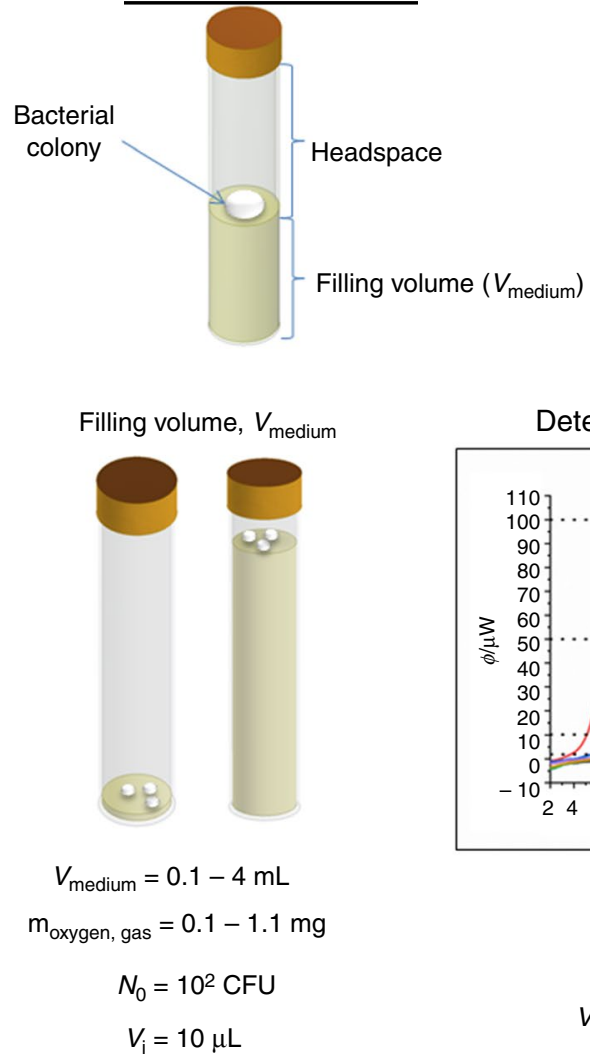

Detection threshold values, $\phi_{\text {dect }}$

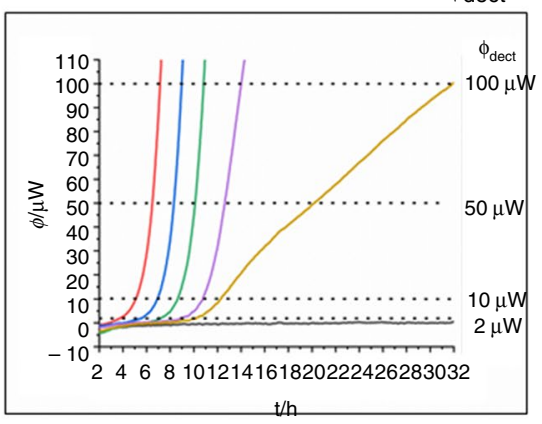

$\phi_{\text {dect }}=2,10,50$ and $100 \mu \mathrm{W}$

$$
\begin{aligned}
N_{0} & =10^{3}-10^{0} \mathrm{CFU} \\
V_{\text {medium }} & =0.1-4 \mathrm{~mL}
\end{aligned}
$$

\section{Liquid Cultivation (SC)}
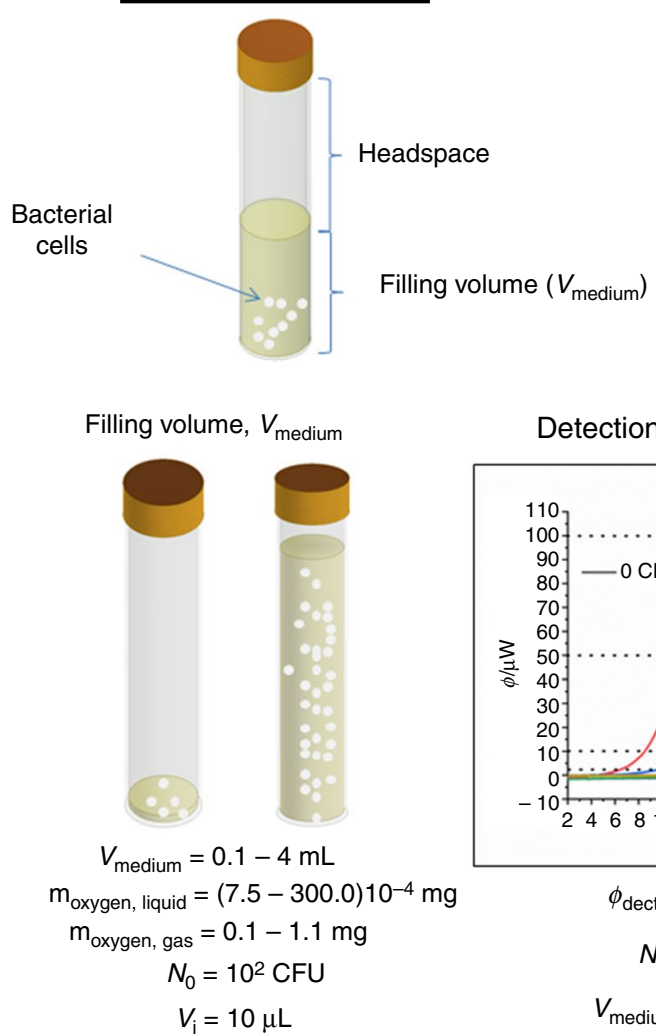

Detection threshold values, $\phi_{\text {dect }}$

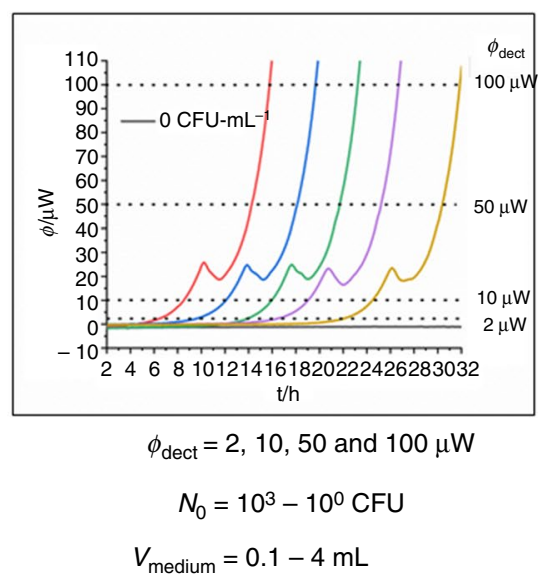


4Fig. 2 Summary of the experimental set-up for IMC experiments to detect bacterial growth. a In solid (SC) and $\mathbf{b}$ in liquid cultivation (LC). Each of the two cultivations is subdivided into two separate experiments: an investigation of the heat flow measurements as a function of the initial number of bacteria $N_{0}$ (in CFU) as well as of the filling volume $V_{\text {medium }}$ (in $\mathrm{mL}$ ). Finally, the measured heat flows are evaluated by different detection threshold values $\Phi_{\text {dect }}$ (in $\mu \mathrm{W}$ )

glass filtration device (diameter $25 \mathrm{~mm}$, Sartorius AG, Göttingen, Germany). Membrane filtration was performed under low pressure. Afterwards, the membrane filters were placed on top of the solid surface or submerged in the liquid medium using sterile tweezers.

Experiments with varying the inoculum volume $V_{\mathrm{i}}$ in LC were performed based on the lowest concentration $\left(c_{0}=10^{1} \mathrm{CFU} \mathrm{\textrm {mL } ^ { - 1 }}\right)$. Different volumes $\left(V_{\mathrm{i}}=0.01\right.$, $0.1,0.25,0.5$ and $1 \mathrm{~mL}$ ) of this concentration (including a sterile blank) were added to $1 \mathrm{~mL}$ DSM-1 medium (results, see $\mathrm{S} 1$ in $\mathrm{SI}$ ).

The second series of IMC experiments dealt with the filling volume $V_{\text {medium }}$ of the calorimetric ampoules. Different filling volumes created different initial conditions concerning the amount of substrate and oxygen (see Fig. 2) so that we were able to investigate the influence on bacterial growth and consequently on the detection of the bacteria. Amounts of oxygen in headspace and liquid phase (see Fig. 2) were calculated based on a mass fraction of oxygen in the air atmosphere of $23.14 \%$ [27] and a concentration of dissolved oxygen in the liquid phase of $7.5 \mathrm{mg} \mathrm{L}^{-1}$ [28] at $30^{\circ} \mathrm{C}$. An inoculum volume of $0.01 \mathrm{~mL}$ of the same concentration $\left(c_{0}=10^{3} \mathrm{CFU} \mathrm{mL} \mathrm{mL}^{-1}\right.$ and including a sterile blank) was added to ampoules which were filled with different volumes of medium $\left(V_{\text {medium }}=0.1\right.$, $0.5,2,3$ and $4 \mathrm{~mL}$ ). In the 4.2-mL ampoules, a residual headspace was thus always present. To evaluate the practical value of the $N_{0^{-}}$and $V_{\text {medium }}$-dependent measured heat flows, they were regarded in the light of different detection threshold values $\left(\Phi_{\text {dect }}=2,10,50\right.$ and $\left.100 \mu \mathrm{W}\right)$.

The last series of experiments were performed in minimal medium (MMKT2440) instead of DMS-1 medium for calorimetric measurements (LC, $n=2$ ). MMKT2440 consisted of (in gL ${ }^{-1}$ ): $\left(\mathrm{NH}_{4}\right)_{2} \mathrm{SO}_{4}(0.5), \mathrm{Na}_{2} \mathrm{HPO}_{4}(0.6)$, $\mathrm{KH}_{2} \mathrm{PO}_{4}(1.4), \mathrm{MgCl}_{2} \cdot \mathrm{H}_{2} \mathrm{O}(0.25), \mathrm{CaCl}_{2} \cdot \mathrm{H}_{2} \mathrm{O}(0.07)$ and glucose $(0.5)$. Again, $1 \mathrm{~mL}$ of the medium and $0.01 \mathrm{~mL}$ of each concentration $\left(10^{5}-10^{1} \mathrm{CFU} \mathrm{mL}^{-1}\right.$, including a sterile blank) were added to $4.2 \mathrm{~mL}$ glass ampoules.

In total, we thus investigated how the initial number of bacteria $N_{0}$, the filling volume of the calorimetric ampoules $V_{\text {medium }}$, the composition of the growth medium as well as detection threshold values $\Phi_{\text {dect }}$ influences the detection of bacterial growth in a $4.2 \mathrm{~mL}$ static ampoule system.

\section{Results and discussion}

\section{Visual detection of colonies as a function of initial bacterial concentration}

The proof of bacterial contaminations in microbiological analysis is still predominated by conventional cultivation mainly on solid culture media $[29,30]$. As our study dealt with the detection time of aerobic bacterial growth using microcalorimetry, a reference value for the detection time of $P$. putida mt-2 KT2440 was determined by CFU counting by five independent observers (see for detailed results S4 in SI). The first appearance of colonies was defined as detection time, and results are shown in Fig. 3. The average CFU detection time of $13.3 \pm 1.3 \mathrm{~h}$ was nearly independent of the initial concentration of bacteria, as expected. The subsequent detection times, determined by IMC experiments, are compared and related to this reference.

\section{Factors influencing the detection time in IMC}

In the following, we define the detection time $t_{\text {dect }}$ as the time required from the insertion of the sample into the IMC instrument until the metabolically determining heat flow signal exceeds statistically significant the noise of the applied IMC. Manufacturers of IMCs specify a limit of detection (LOD), but in practice, however, a fixed value for the detectable heat flow $\Phi_{\text {dect }}$ (detection threshold value) of the respective instrument is typically defined empirically.

On this occasion, it is important to mention that our study aims at the earliest detection of bacterial growth; thus, our considerations are restricted to exponential growth at the beginning of the metabolically determining heat flow. In the following, we assume that the heat output of a growing culture starting from an initial cell number $N_{0}$ is determined by the specific growth rate $\mu$ (in $\mathrm{h}^{-1}$ ) of an exponentially growing culture [26], and by a mean cell-specific heat production rate $\varphi_{0}$ (in $\mathrm{W}$ ) $[17,21]$ (see both equations in Fig. 4). At this point, we have to differentiate between two scenarios: (1) at $t=0$, the sample already contains so many bacteria that the actual metabolic heat production rate exceeds the detection threshold value $\Phi(t) \geq \Phi_{\text {dect }}$ or (2) at $t=0$, the sample contains so few bacteria that the metabolic heat production is below the detection threshold value and heat can only be detected after exponential growth (if sufficient nutrients are available). The heat production rate will rise a detectable heat flow at time $t=t_{\text {dect }}$. Equation (1) relates the detection time $t_{\text {dect }}$ (in $\mathrm{h}$ ) and the detectable heat flow $\Phi_{\text {dect }}$ (in W).

$t_{\text {dect }}=\frac{\ln \left(\frac{\Phi_{\text {dect }}}{\varphi_{0}}\right)-\ln \left(N_{0}\right)}{\mu}$ 


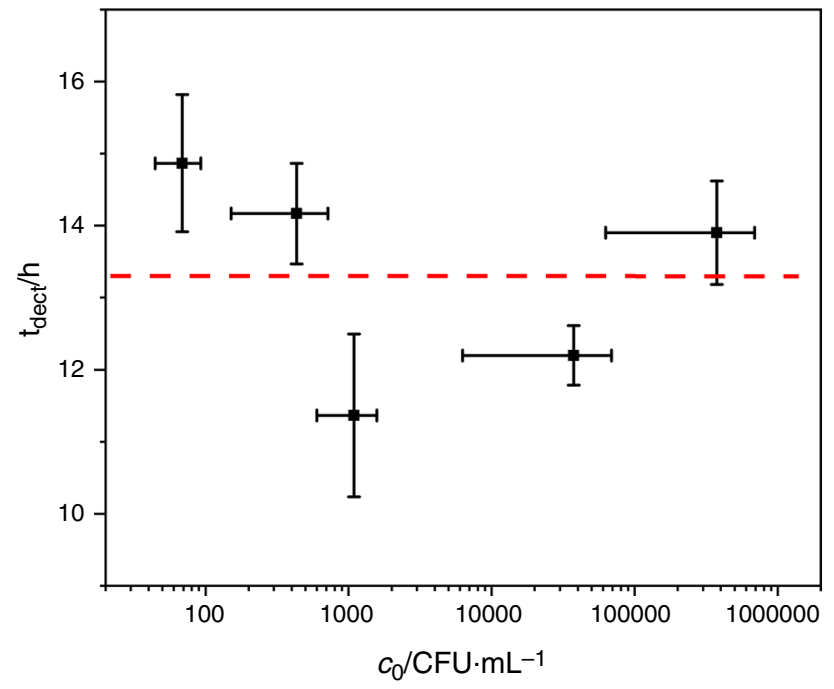

Fig. 3 Detection times $t_{\text {dect }}$ as a function of the initial concentration of bacteria $c_{0}$ obtained. The red dash line represents the average CFU detection time. (Color figure online)

The meanings and derivation of the variables are given in Fig. 4.

In principle, preparative, technical and biological factors of influence on the detection time can be distinguished. These factors will be examined in the following.

\section{Calorimetric detection time as a function of sample preparation and inoculum size}

The initial number of bacteria $N_{0}$ (in CFU) can be influenced by sample preparation. Compared with classical CFU counting, where the inoculum size does not affect the time needed for detection of colonies (see Fig. 1 and S1 in SI), IMC detection depends on $N_{0}$ according to Eq. (1). Short detection times require high $N_{0}$ values which in principle can be achieved in two ways. First, the sample volume added for inoculation can be increased. However, this is generally restricted by the small size of common calorimetric vessels (typically in the range from 4 to $125 \mathrm{~mL}$ ). In the case of LC, inoculum volumes of $>1 \mathrm{~mL}$ are applicable but for SC the upper limit even in large calorimetric vessels is approx. $1 \mathrm{~mL}$ because of their relatively small cross-sectional areas. Second, $N_{0}$ can be increased from a larger volume, for example by membrane filtration (MF). The reduction of IMC detection time by MF enrichment was recently shown with bacteria (i.e. L. plantarum) grown anaerobically in SC [25]. In principle, there are two common cultivation techniques (SC and LC) which will be investigated in the following.

\section{Solid cultivation (SC)}

The initial number of bacteria $\left(N_{0}\right)$ dependency of heat flow measurements obtained on solid medium is shown in Fig. 5. All heat flow signals could be roughly divided into five stages: I. baseline, II. exponential (high $N_{0}$ ) or linear (low $N_{0}$ ) increase in heat flow, III. maximum heat flow, IV. decreasing heat flow and V. return to the baseline. All heat flow signals consisted of a single peak of varied shape. Heat flow signals were broader when $N_{0}$ was lower (Fig. 5a). Comparing these measurements with literature data revealed that other bacteria (Lactobacillus plantarum [25], Mycobacterium species [23] and E. coli [24]) displayed similar heat flow pattern on solid medium.

Shape and amplitude of the heat flow signals depend on the inocula size (i.e. initial number of bacteria). Larger $N_{0}$ consumed substrate and oxygen faster; thus, the bacteria
Fig. 4 Factors influencing the time needed to detect bacterial contaminations by IMC measurements

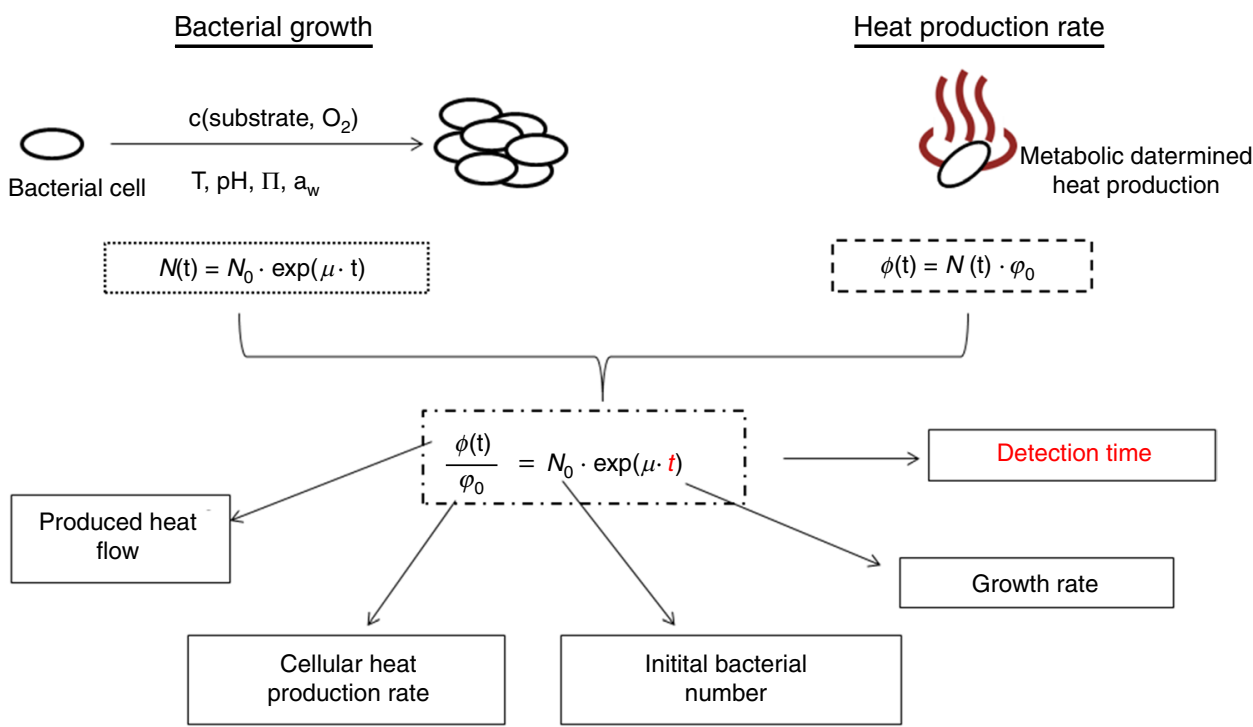




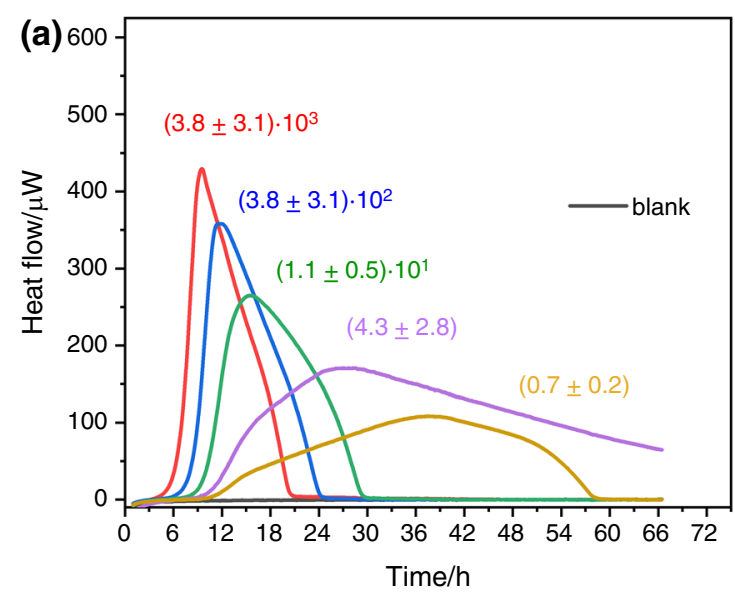

Fig. 5 IMC experiments performed on SC. The $N_{0}($ mean $\pm \mathrm{SD})$ in $\mathrm{CFU}$ are indicated next to the heat flow signals and based on the inoculum volume $(10 \mu \mathrm{L})$. a Dependency of heat traces on the $N_{0}$. b Mag-

reached early the maximum specific growth rate and consequently produced a larger heat flow (see Fig. 5a). This finally results in a sharper peak. Independent of $N_{0}$, the average total amount of heat released was $(12.0 \pm 0.6) \mathrm{J}$. This value was confirmed by using the oxycaloric equivalent calculated for aerobic growth (see for details chapter S3.1 in SI). The total heat produced by oxygen-limited aerobic bacteria should thus be in the same range irrespective of species and inoculum size.

Following Eq. (1), the metabolically determining heat flow signals were detected earlier when the $N_{0}$ was higher (Fig. 5b). BRAISSANT and co-workers performed bacterial concentration-dependent IMC experiments with Mycobacterium tuberculosis and observed similar results [23]. For the determination of the detection time, a detection threshold value of $2 \mu \mathrm{W}$ was assumed for TAM III. This is 10 times higher than the instrument noise specified by the manufacturer (www.tainstrument.com). The resulting detection times are summarized in Table 1.

\section{Liquid cultivation (LC)}

The $N_{0}$ dependency of heat flows measured for LC are shown in Fig. 6. Here, six stages in all heat flow signals can be distinguished: (1) baseline, (2) a first exponential increase in heat flow, (3) a slight decrease (a phase being absent for SC), (4) a second exponential increase, (5) maximum in heat

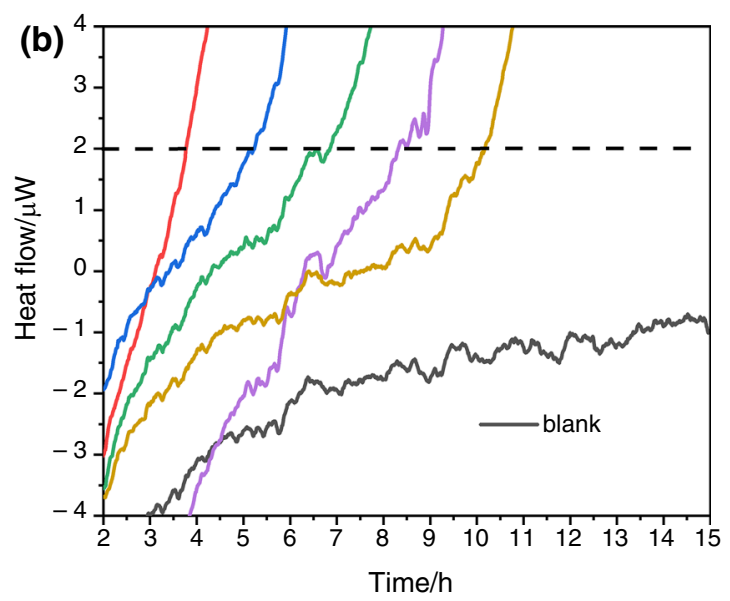

nification of heat flow signals near the detection zone, the dotted line representing the predefined detection threshold value of $2 \mu \mathrm{W}$

flow and (6) return to baseline level (Fig. 6a). This heat flow pattern was also observed by BONKAT and co-workers for fast-growing mycobacteria Mycobacterium smegmatis and Mycobacterium phlei [31]. All heat flow signals had two peaks, a small initial one with a magnitude of approx. $25 \mu \mathrm{W}$ followed by a large one of approx. $500 \mu \mathrm{W}\left(500 \mathrm{~mW} \mathrm{~L}^{-1}\right)$. The first peak can be explained by the consumption of oxygen dissolved in liquid medium (approx. $23.4 \mu \mathrm{mol}$ at $30^{\circ} \mathrm{C}$ ) [28]. Using the oxycaloric equivalent $\left(-455 \mathrm{~kJ} \mathrm{~mol}^{-1} \mathrm{O}_{2}\right)$ [32], this corresponds to a heat production of approx. $0.1 \mathrm{~J}$. This value was confirmed by integrating the heat flow curve up to the maximum of the first peak (see chapter S3.2 in SI).

The second peak might reflect dynamics of substrate or oxygen depletion. Unlike, the different times of onset, the form of the heat flow signals was independent of $N_{0}$ variation making LC more promising for the detection of low $N_{0}$ than SC. The different growth behaviour of the bacteria $\mathrm{LC}$ versus $\mathrm{SC}$ determines the shape of the heat flow signal. A bacterial colony relies on substrate (or oxygen) diffusing towards the substratum-colony interface, whereas in LC, bacteria are surrounded by dissolved substrate and oxygen, though provision with oxygen may be limited by diffusion from the headspace.

As expected from Eq. (1), the onset of heat flow signals depended on $N_{0}$ (Fig. 6b). The detection time was determined in the same way as for SC and is given in Table 1. Interestingly, cultivation in liquid or on solid medium (using
Table 1 Summary of the detection times (mean \pm SD) determined at a detection threshold value of $2 \mu \mathrm{W}$

\begin{tabular}{llllll}
\hline $\begin{array}{l}\text { Growth } \\
\text { condition }\end{array}$ & $\begin{array}{l}t_{\text {dect }} / \mathrm{h} \\
3.8 \times 10^{3} \mathrm{CFU}\end{array}$ & $\begin{array}{l}t_{\text {dect }} / \mathrm{h} \\
3.8 \times 10^{2} \mathrm{CFU}\end{array}$ & $\begin{array}{l}t_{\text {dect }} / \mathrm{h} \\
1.1 \times 10^{1} \mathrm{CFU}\end{array}$ & $\begin{array}{l}t_{\text {dect }} / \mathrm{h} \\
4.3 \mathrm{CFU}\end{array}$ & $\begin{array}{l}t_{\text {dect }} / \mathrm{h} \\
0.7 \mathrm{CFU}\end{array}$ \\
\hline $\mathrm{SC}$ & $(3.9 \pm 0.1)$ & $(5.4 \pm 0.2)$ & $(7.2 \pm 0.3)$ & $(8.7 \pm 0.3)$ & $(10.5 \pm 0.4)$ \\
$\mathrm{LC}$ & $(3.9 \pm 0.1)$ & $(5.6 \pm 0.1)$ & $(7.8 \pm 0.1)$ & $(9.4 \pm 0.2)$ & $(11.1 \pm 0.6)$ \\
\hline
\end{tabular}




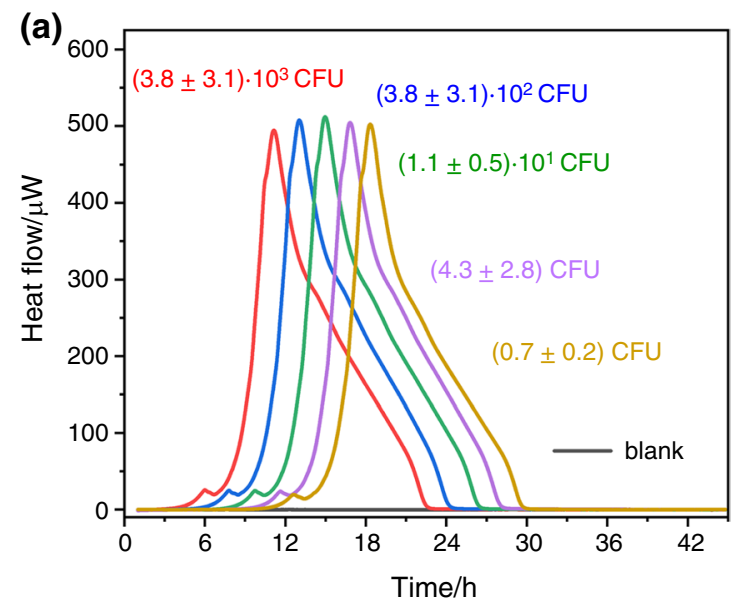

Fig. 6 IMC experiments performed in LC. The $N_{0}($ mean $\pm \mathrm{SD})$ in CFU are indicated next to the heat flow signals and based on the inoculum volume $(10 \mu \mathrm{L})$. a Dependency of heat traces on the $N_{0}$. b Mag-

the same inoculum volume of $10 \mu \mathrm{L}$ ) did not influence the detection time much at the predefined detection threshold value of $2 \mu \mathrm{W}$. For such a low heat flow, the provision of substrates and oxygen does not limit the growth. However, to achieve the earliest possible detection time, LC offers the major advantage that the inoculum volume can easily adapt to the overall volume of the calorimetric vessel.

Based on the CFU detection time determined by visual inspection $(13.3 \pm 1.3 \mathrm{~h})$ of bacterial growth on solid medium, we could show with our results that a faster detection by IMC is possible. High $N_{0}\left((3.8 \pm 3.1) \times 10^{3} \mathrm{CFU}\right)$ were detectable after $3.9 \mathrm{~h}$ for both on solid as well as in liquid medium and low $N_{0}(0.7 \pm 0.2 \mathrm{CFU})$ after $10.6 \mathrm{~h}$ for solid medium and $11.1 \mathrm{~h}$ for liquid medium (considering a detection threshold value of $2 \mu \mathrm{W}$ ). Furthermore, if one compares the error of the detection times, it became apparent that calorimetric detections have a smaller error independent of $N_{0}$. This difference can be attributed to the subjective evaluation of colony detection in the case of visual inspection. Similar conclusions were drawn during the visual inspection of pharmaceutical products utilizing sterility testing [16]. A summary of all $N_{0}$-dependent heat flow signals measured on $\mathrm{SC}$ and $\mathrm{LC}$ is given in chapter S5 in the SI.

\section{Enrichment via membrane filtration (MF)}

One possibility to increase $N_{0}$ and to shorten detection is the enrichment from a larger sample volume using membrane filtration (MF). Depending on the background of the analysed sample, $N_{\mathrm{o}}$ can range from a few bacteria cells (e.g. drinking water) to more than $10^{6}$ (e.g. foodstuff) per $\mathrm{mL}$ sample volume [33]. Using the MF process thus larger sample volumes (e.g. $10 \mathrm{~mL}$ instead of $0.01 \mathrm{~mL}$ ) could be subjected to calorimetric measurements. Figure 7

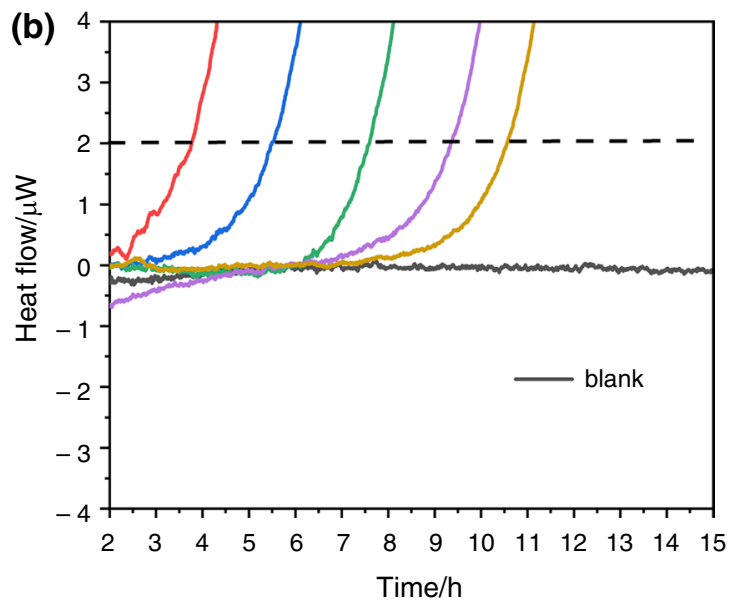

nification of heat flow signals near the detection zone, the dotted line representing the predefined detection threshold value of $2 \mu \mathrm{W}$

compares the heat flow signals with and without MF for SC and LC (taken from Figs. 5a, 6a).

Considering the enrichment factor of 1000 , the obtained heat flow signals showed two substantial changes. Firstly, an increase in $N_{0}$ led to a reduction in detection time (Fig. 7b, d) independently of the detection threshold value $(10,50 \mu \mathrm{W})$. The highest $N_{0}$ (solid red curve) corresponded probably to an $N_{0}$ of approx. $10^{6}$ (assumption based on the enrichment factor of 1000 and an initial number of approx. $10^{3}$ bacteria in $0.01 \mathrm{~mL}$ inoculum volume) in the filter produced an initial heat flow of $25 \mu \mathrm{W}$. These results are very consistent with literature assumptions that 10.000 to 100.000 active bacteria are necessary to produce a heat flow above the limit of thermal detection (LOD $=0.2 \mu \mathrm{W}$ for the TAM III, www.tains truments.com) [34]. Secondly, the shape of the heat flow signals varied strongly between SC (Fig. 7a) and LC (Fig. 7c). Interestingly, the shape of the heat flow signals obtained on SC with (solid curves) and without (dash curves) MF varied especially for the lowest $N_{0}$ (yellow curves). In theory, the heat flow curves with the highest $N_{0}$ (red and blue dashed curves) contain the same number of bacteria as the two curves with the lowest $N_{0}$ (violet and yellow solid curves) due to MF. Consequently, curves should coincide. However, there is some small variation. Small inaccuracies in the placement of the filter on the agar surface and the consequence that not all bacteria have same access to the solid medium could be the cause. In the case of LC, the two heat flow signals with the same $N_{0}$ (red and blue dash curves, and violet and yellow solid curves, Fig. 7d) overlapped. This may indicate that the bacteria were able to detach themselves from the filter surface and access the substrates and oxygen via free movement in liquid phase. The increase in turbidity after the measurement confirmed this assumption. 

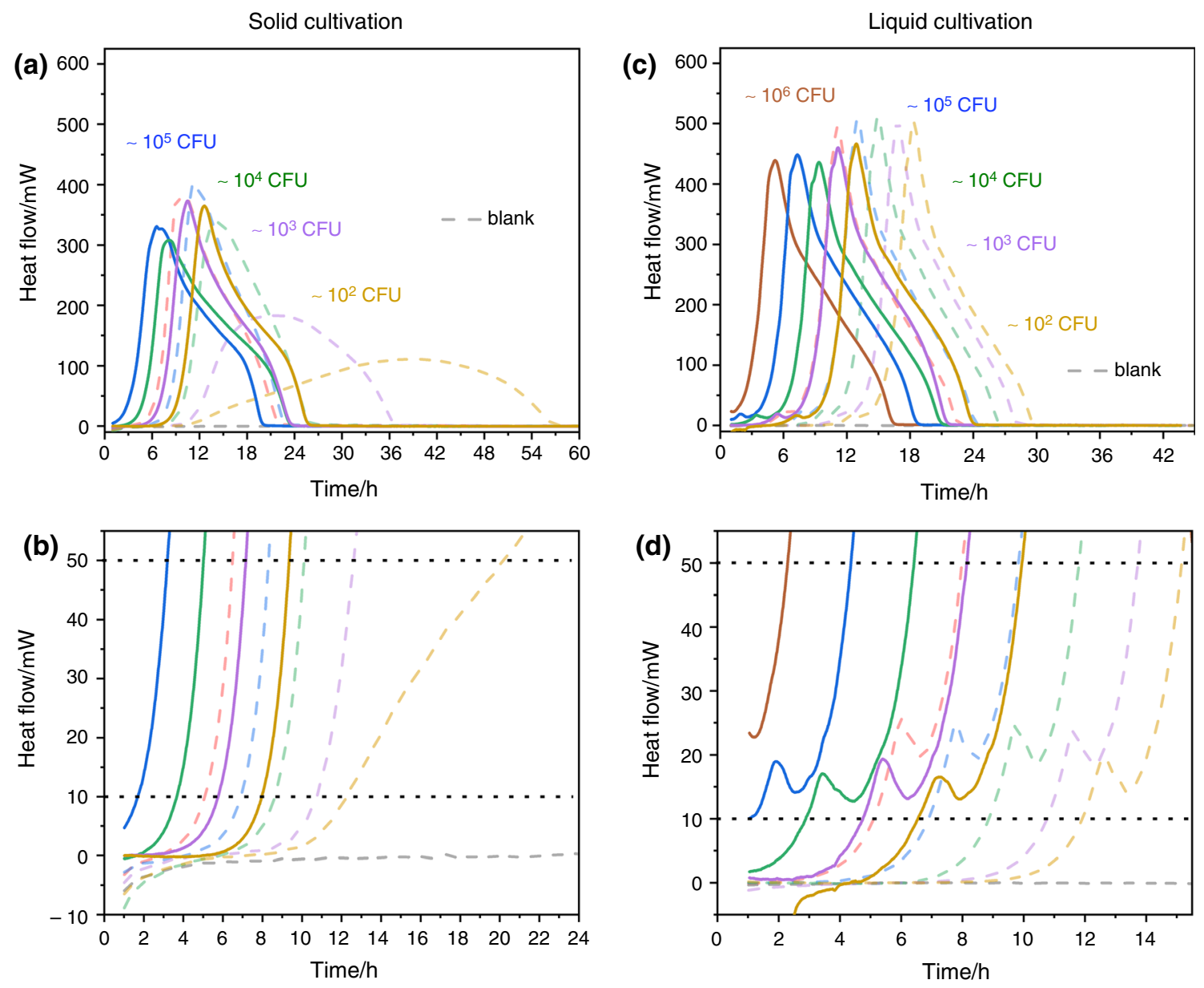

Fig. 7 IMC experiments performed after enrichment by MF. a Comparison of heat flow signals on SC with MF (solid curves) and without MF (dashed curves, approximated $N_{0}$ in CFU are indicated next to the heat flow signals and based on the filtration volume, $10 \mathrm{~mL}$ ). b Magnification of the heat flow signals (SC) near the detection thresh-

It is important to mention here that membrane filtration is accompanied by a certain sample preparation time, and this should be taken into account for detection. However, there is also an argument for membrane filtration. For slowly growing bacteria, the time gained through enrichment can compensate for the loss through sample preparation time.

\section{Influence of IMC performance on the detection time}

\section{Influences of technical specifications}

The detectable heat flow $\Phi_{\text {dect }}(t)$ is mainly determined by the accuracy (lowest resolution in temperature difference which can still convert into voltage) of the detector, a thermoelectric generator (TEG), and the temperature constancy in the entire system. The TEG consists of several serially connected thermocouples. The most common microcalorimeters used to investigate cellular systems are based on

old values 10 and $50 \mu \mathrm{W}$. c Comparison of heat flow signals in LC with MF (solid curves) and without MF (dashed curves). d Magnification of the heat flow signals (LC) near the detection threshold values 10 and $50 \mu \mathrm{W}$

the principle of heat conduction and work in isothermal operation mode [8]. The aim is to ensure that as much of the metabolic heat as possible flows through the detector (TEG). Mathematically, this can be expressed as follows [35]:

$\Phi(t)=\kappa \cdot n_{\mathrm{TEG}} \cdot \Delta T$

where $\kappa\left(\right.$ in $\left.\mathrm{W} \mathrm{K}^{-1}\right)$ is the thermal conductivity of a thermocouple, $n_{\mathrm{TEG}}$ the number of thermocouples and $\Delta T$ (in K) the temperature difference between the upper and lower surface of the TEG. The voltage $U(t)$ generated at the TEG can be described by Eq. (3) [35]:

$U(t)=\alpha \cdot n_{\mathrm{TEG}} \cdot \Delta T$

where $\alpha$ (in $\mathrm{V} \mathrm{K}^{-1}$ ) is the Seebeck coefficient of the thermocouples. According to the difference in temperature between the upper surface (increase in temperature due to metabolic heat caused by bacteria) and the lower surface (constant 
temperature of the heat sink given by the system), a voltage is generated (Seebeck effect). In other words, the heat conducted via the TEG $\Phi(t)$ is proportional to the measured voltage $U(t)($ Eq. 4) [36]:

$\Phi(t)=\varepsilon \cdot U(t)$

The proportionality factor $\varepsilon$ (in $\mathrm{W} \mathrm{V}^{-1}$ ), also referred to as the calibration constant, is composed as follows:

$\varepsilon=\frac{\kappa}{\alpha}$

Since these are two material constants, there are possibilities to influence this quotient and to keep the value of $\varepsilon$ as small as possible. A low specific thermal conductivity and a large Seebeck coefficient are aimed for [37].

As already mentioned, the bottom side of the TEG is kept constant by a temperature-controlled heat sink. Due to the small temperature increase caused by the metabolic heat of the growing bacterial cultures [9], already small temperature fluctuations on the heat sink of the instruments can have a direct effect on the performance of the IMC measurement. As a result of such fluctuations, noise or drifts may superpose the metabolic heat flow signals and a delayed detection time would be determined.

Interestingly, most applications on bacteria detection by IMC in literature were performed in $4 \mathrm{~mL}$ static ampoules using high-performance microcalorimeters (low detection thresholds are applicable) [15, 19, 22, 23, 31]. In calorimeters with a high detection threshold, this deficit is typically compensated by large sample volumes containing more heat-producing bacteria [38]. However, due to the larger sample volume, only a few simultaneous measurements are possible. For the practitioner, however, in addition to short detection times and simple calorimeters, the number of simultaneous measurement possibilities is also decisive. Thus, it is a big difference to measure up to 48 samples in a high-performance calorimeter or up to 12 samples using a simple calorimeter having the same volume-related limit of detection or to measure up to 48 samples in a simple calorimeter with a lower limit of detection as long as the bacterial contamination is detected early enough. To address these issues, we simulated the effect of calorimeter detection thresholds as well as the sample volumes on the detection time directly, assuming different empirical threshold values $\left(\Phi_{\text {dect }}=2,10,50\right.$ and $\left.100 \mu \mathrm{W}\right)$ in the context of a static 4-mL ampoule system. These assumptions are supported by manufacturer information and literature data and selected to cover almost all commercially available IMC devices used for biocalorimetry (see Table 2).

\section{Influence of the initial number of bacteria on the detectable heat flow}

To relate detectable heat flows to common microcalorimeters (see Table 2), detection threshold values $(2,5,10,50$ and $100 \mu \mathrm{W}$ ) were used to establish detection times for LC and $\mathrm{SC}$ in conventional static 4-mL glass ampoules. Figure 8 summarizes the results. For the sake of simplicity, only the results obtained with the highest (red curves) and the lowest $N_{0}$ (yellow curves) are discussed.

Two cases will be considered: first, the difference between high and low $N_{0}$ at different detection threshold values and different cultivation modes (LC vs. SC) and second, the influence of different detection threshold values at constant $N_{0}$.

The difference in the detection time between highest (red curve) and lowest (yellow) $N_{0}$ was independent of the detection threshold for LC (approx. $7 \mathrm{~h}$ ). For SC, the same delay was observed at detection thresholds $\leq 10 \mu \mathrm{W}$ (Fig. 8a). Whereas the difference in detection times at a threshold value of $50 \mu \mathrm{W}$ was already twice as long (approx. $13 \mathrm{~h}$ ) and at $100 \mu \mathrm{W}$ almost four times as long (approx. $26 \mathrm{~h}$ ).

Table 2 Typical detection threshold values for IMC instruments used in biocalorimetry

\begin{tabular}{|c|c|c|c|c|c|}
\hline Calorimeter & Manufacturer & $\begin{array}{l}\text { Empirical thresh- } \\
\text { old/ } \mu \mathrm{W}\end{array}$ & $\begin{array}{l}\text { Filling volume of } \\
\text { ampoule/mL }\end{array}$ & $\begin{array}{l}\text { Volume-specific } \\
\text { threshold } / \mathrm{mW} \mathrm{L}^{-1}\end{array}$ & References \\
\hline TAM 48 & TA instruments & 2 & n.a. & - & {$[23]$} \\
\hline TAM III & & 10,2 & $3,0.2$ & 3,10 & {$[19,25]$} \\
\hline TAM air & & 25 & $7-5$ & $4-5$ & {$[38]$} \\
\hline MC-Cal/100 P & $\begin{array}{l}\text { C3 Prozess- und Analysen- } \\
\text { technik GmbH }\end{array}$ & $100^{b}, 28^{c}$ & $1^{\mathrm{b}}, 0.4^{\mathrm{c}}$ & $100^{\mathrm{b}}, 70^{\mathrm{c}}$ & [39] \\
\hline BioCal 2000 & Calimetrix Inc. & 100 & n.a. & - & www.calmetrix.com \\
\hline calScreener & Symcel AB & 1 & 0.3 & 3 & [40] \\
\hline
\end{tabular}

n.a. not available

${ }^{\mathrm{a}}$ Calculated by using the filling volume of the ampoules

${ }^{\mathrm{b}}$ Determined in our study

${ }^{\mathrm{c}}$ Calculated from the available data 

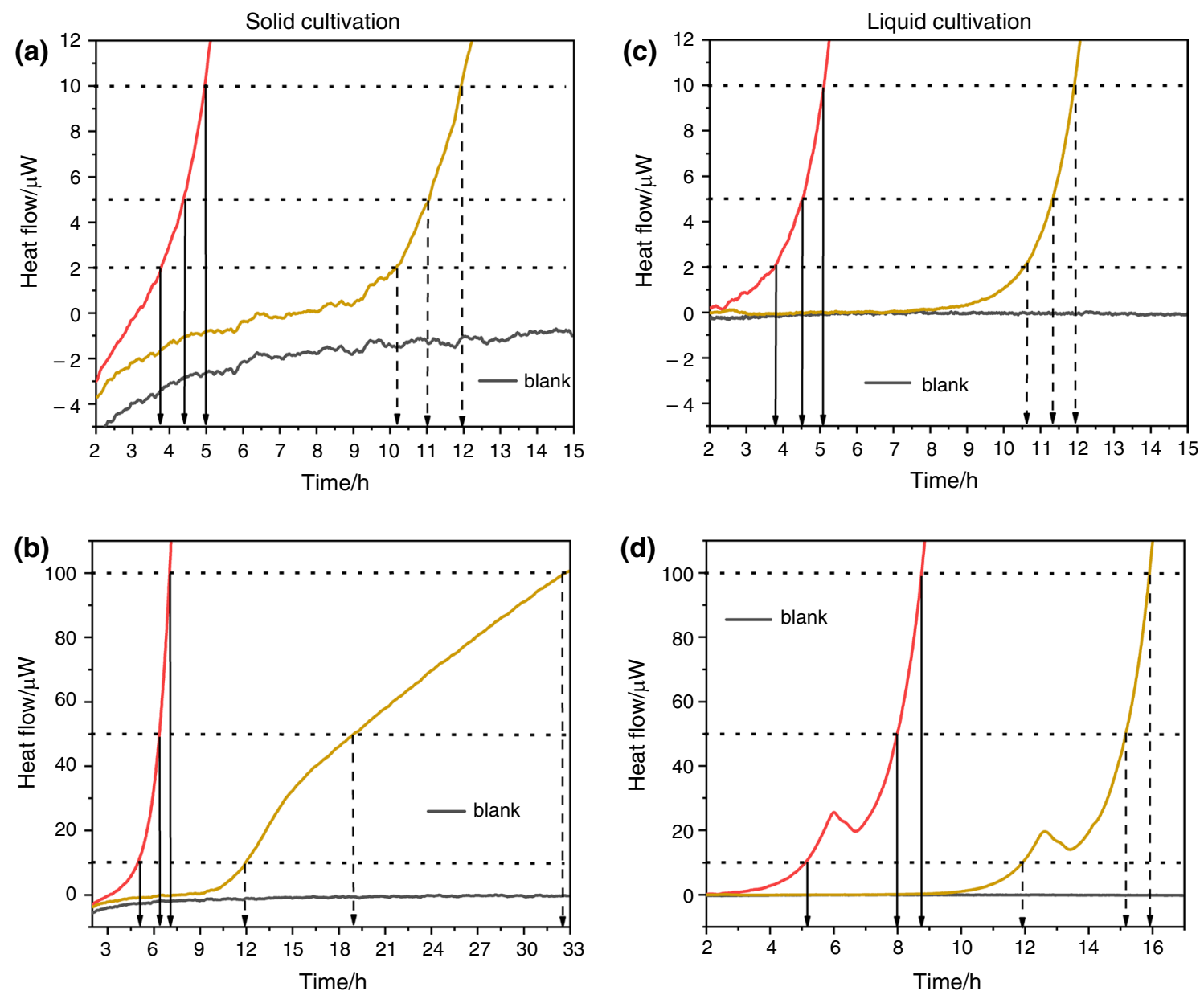

Fig. 8 Influence of simulated detection thresholds on detection times achieved in IMC experiments depending on $N_{0}$ in solid $(\mathbf{a}, \mathbf{b})$ as well as in liquid (c, d) medium. Red: $(3.8 \pm 3.1) \cdot 10^{3}$ CFU. Yellow: $(0.7 \pm 0.2)$ CFU. a Magnification of the heat flow signals measured in SC near detection threshold value, the dotted lines representing $\Phi_{\text {dect }}$

Considering now a constant $N_{0}$, the heat flow signals showed that, regardless of the cultivation mode (solid or liquid), the detection time between high-performance microcalorimeters (threshold values of 2 to $10 \mu \mathrm{W}$ ) varied by roughly $2 \mathrm{~h}$ following Eq. (1) (Fig. 8a, c). The exponential increase in the heat flow confirms the assumption of exponential growth. At high $N_{0}$ (red curve), the delay in detection with $100 \mu \mathrm{W}$ versus $2 \mu \mathrm{W}$ was $3 \mathrm{~h}$ with SC (Fig. 8a, c) and was $5 \mathrm{~h}$ with LC (Fig. 8c, d). The difference in detection time of $2 \mathrm{~h}$ between LC and SC might be caused by the influence of oxygen diffusion on the metabolic response of $P$. putida mt-KT2440 [41]. The growth process slows down under conditions influenced by diffusion. Simulations [42] point to the special role of oxygen diffusion in static, closed ampoules.

At low $N_{0}$ (yellow curve), the delay in detection with threshold values of $100 \mu \mathrm{W}$ versus $2 \mu \mathrm{W}$ increased by $22 \mathrm{~h}$ with SC (Fig. 8a, b) and by $5 \mathrm{~h}$ with LC (Fig. 8c, d). The

of 2, 5 and $10 \mu \mathrm{W}$. b Heat flow signals measured in SC near $\Phi_{\text {dect }}$ of 10,50 and $100 \mu \mathrm{W}$. c Magnification of the heat flow signals measured in LC near detection threshold value, the dotted lines representing $\Phi_{\text {dect }}$ of 2, 5 and $10 \mu \mathrm{W}$. d Heat flow signals measured in LC near $\Phi_{\text {dect }}$ of 10,50 and $100 \mu \mathrm{W}$. (Color figure online)

difference in LC has already been discussed. However, a completely different picture is obtained at low $N_{0}$ in the case of SC. The difference in detection time of $22 \mathrm{~h}$ was caused by a change from exponential to substrate diffusion-limited linear growth at about $30 \mu \mathrm{W}$ (or after $14 \mathrm{~h}$ ). This growth behaviour is characteristic for the formation of bacterial colonies on solid substrate [43,44]. This effect was also reflected in the size of the colonies formed (see for details SI).

Finally, to confirm the detection times simulated here, we used a less powerful cement calorimeter (LOD of $20 \mu \mathrm{W}$ for the MC-Cal/100 P, https://www.c3-analysentechnik.de) to perform the same $N_{0}$-dependent measurements in LC $(n=2)$ under the same conditions as described in Fig. $2 \mathrm{~b}$. The detection times are summarized in Table 3 , and the corresponding heat flow signals are given in chapter S6 in the SI.

If we now compare the detection times in Table 3 (detection threshold $100 \mu \mathrm{W}$ ) with the CFU detection time 
Table 3 Comparison of detection times determined with two different calorimeters at a detection threshold value of $100 \mu \mathrm{W}$

\begin{tabular}{lllllc}
\hline Calorimeter & $t_{\text {dect }} / \mathrm{h}$ & $t_{\text {dect }} / \mathrm{h}$ & $t_{\text {dect }} / \mathrm{h}$ & $t_{\text {dect }} / \mathrm{h}$ & $t_{\text {dect }} / \mathrm{h}$ \\
& $3.8 \times 10^{3} \mathrm{CFU}$ & $3.8 \times 10^{2} \mathrm{CFU}$ & $1.1 \times 10^{1} \mathrm{CFU}$ & $4.3 \mathrm{CFU}$ & $0.7 \mathrm{CFU}$ \\
\hline TAM III $^{\mathrm{a}}$ & $(8.8 \pm 0.1)^{\mathrm{c}}$ & $(10.7 \pm 0.1)$ & $(12.6 \pm 0.1)$ & $(14.5 \pm 0.1)$ & $(16.1 \pm 0.5)$ \\
$\mathrm{MC}-C a 1 / 100 \mathrm{P}^{\mathrm{b}}$ & 9.1 & 11.6 & 13.5 & 15.4 & 16.4 \\
& 8.9 & 11.8 & 13.7 & 15.8 & n.d. \\
\hline
\end{tabular}

n.d. not detected

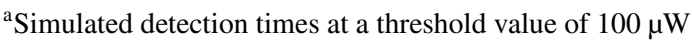

${ }^{\mathrm{b}}$ Only measured in duplicates

${ }^{\mathrm{c}}$ Mean \pm SD
$(13.3 \pm 1.3)$ determined by visual inspection, it must be concluded that if only less than ten bacteria cells are presented at the beginning of the measurement, visual inspection might be slightly faster. However, the time needed to form all countable colonies on a plate that are visible for the naked eye is mostly higher.

\section{Influence of the filling volume of medium on the detectable heat flow}

To investigate the influence of oxygen and substrate on the detection time being a function of the detectable heat flow, IMC experiments were performed with different filling volumes of liquid and solid medium $\left(V_{\text {medium }}\right)$ in the calorimetric ampoules. The change in $V_{\text {medium }}$ resulted in different amounts of oxygen and substrate, which are available to the bacteria for growth. Due to the close relation between bacterial growth and metabolic heat according to Eq. (1), we expect an influence on detection time, especially in LC. Here, the metabolic response of the bacteria to oxygenlimiting conditions should be more noticeable. But first, we examine the heat flow signals recorded at different $V_{\text {medium }}$

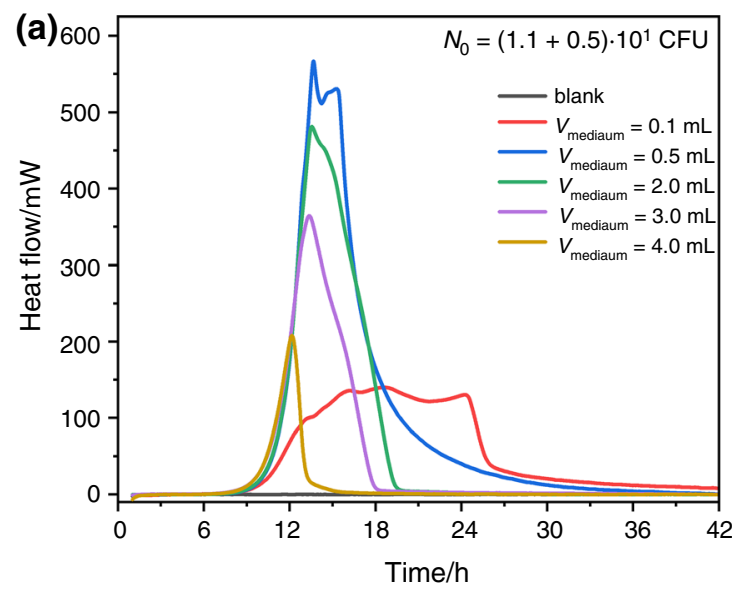

Fig. 9 Influence of $V_{\text {medium }}$ on the heat flow signals obtained from IMC experiments conducted on solid medium. a Heat flow traces depending on $V_{\text {medium }}$. b Relationship between detection time and on solid medium (Fig. 9). Both factors, substrate and oxygen quantity, influenced the growth of bacteria on solid medium. At low filling volumes $\left(V_{\text {medium }}=0.1 \mathrm{~mL}\right)$, the bottom of the ampoule was not completely and evenly covered by the medium as well as by $V_{\mathrm{i}}$; hence, the shape of the heat flow curve (red curve, Fig. 9a) differed in comparison to the other.

If one compares only the increase of the heat flow curves in Fig. 9a considering detection threshold values, all heat flows show exponential shape (except for the red curve). Depending on the total amount of oxygen (determined by $\left.V_{\text {medium }}\right)$, the maximum heat flow varied and reached at $V_{\text {medium }}=0.5 \mathrm{~mL}$ (blue curve) a maximum value of $575 \mu \mathrm{W}$ $\left(1150 \mathrm{~mW} \mathrm{~L}^{-1}\right)$. Detection times were the same for different $V_{\text {medium }}$ regarding constant threshold value (Fig. 9b). However, decreasing performance of the instrument caused later detection, regardless of $V_{\text {medium }}$ (Fig. 9b). The difference in detection time at threshold values of 2 and $100 \mu \mathrm{W}$ was approx. $4 \mathrm{~h}$ and almost independently of the respective $V_{\text {medium }}$.

The shape of heat flows obtained from $V_{\text {medium }}$-dependent experiments in LC (Fig. 10) showed a stronger variation compared with those obtained from SC. To describe the

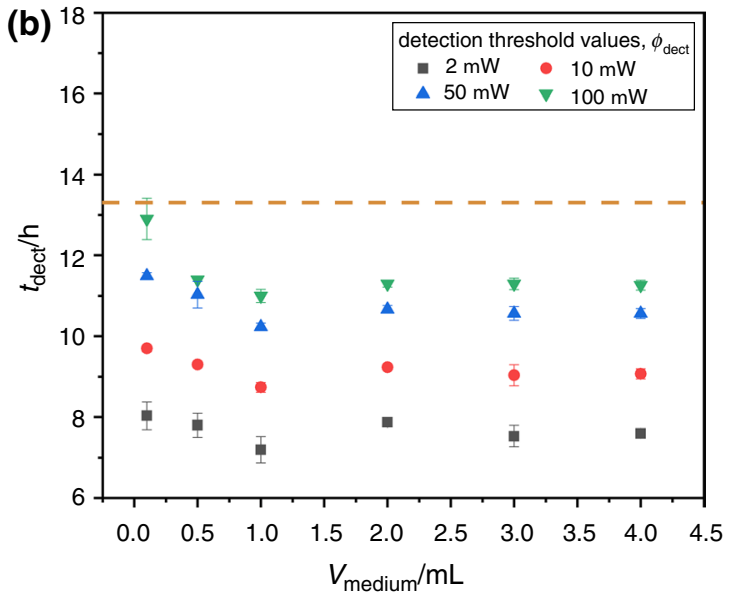

$V_{\text {medium }}$ as a function of different detection threshold values $\Phi_{\text {dect }}=2$, 10,50 and $100 \mu \mathrm{W}$. For comparison, the dashed line in B indicates the mean CFU detection time determined by visual inspection 
shape of the heat flow curves, we have to distinguish between dissolved oxygen in the liquid phase which is immediately available to the bacteria, and oxygen in the gas phase which is only available to the bacteria through slow transport from gas phase into liquid phase and thus with delay [42]. The initial ratio between dissolved and unsolved oxygen was determined by $V_{\text {medium }}$ of the liquid. Besides, a higher $V_{\text {medium }}$, as mentioned before, led to an overall decrease in the total amount of oxygen in the calorimetric ampoule (minimum and maximum oxygen quantities are shown in Fig. 2b). The lowest filling volume $\left(V_{\text {medium }}=0.1 \mathrm{~mL}\right)$ showed a slightly different heat flow signal (Fig. 10a, red curve) compared to the others. Possibly the interplay between substrate and oxygen limitation changed the metabolic response of the bacteria, thus causing a diffuse heat flow signal was obtained (Fig. 10a, red curve). Detection times were the same for different $V_{\text {medium }}$ at threshold values of 2 and $10 \mu \mathrm{W}$ (black and red points, Fig. 10b). Additionally, average detection times were in the same magnitude $7.5 \mathrm{~h}$ at $2 \mu \mathrm{W}$ and $9.5 \mathrm{~h}$ at $10 \mu \mathrm{W}$ for both SC and LC (Figs. 9b, 10b). The reason for this was that at time of these detectable heat flows sufficient oxygen and substrate were available to the bacteria. This corresponds to our assumption of a constant cell-specific heat production rate.

However, instruments with a worse threshold value $(>10 \mu \mathrm{W})$ led to later detection but unlike SC, a $V_{\text {medium }^{-}}$ dependent behaviour was observed (Fig. 10b). At a detection threshold value of $100 \mu \mathrm{W}$ (green points, Fig. 10b), an almost linear relationship between the detection time and $V_{\text {medium }}$ was observed. An assumption for this might be the extended diffusion path (caused by an increase in $V_{\text {medium }}$ ) for oxygen which diffused by transport from gas phase into liquid phase and the bacteria freely moving in medium. This would also explain the shift of the second peak depending on

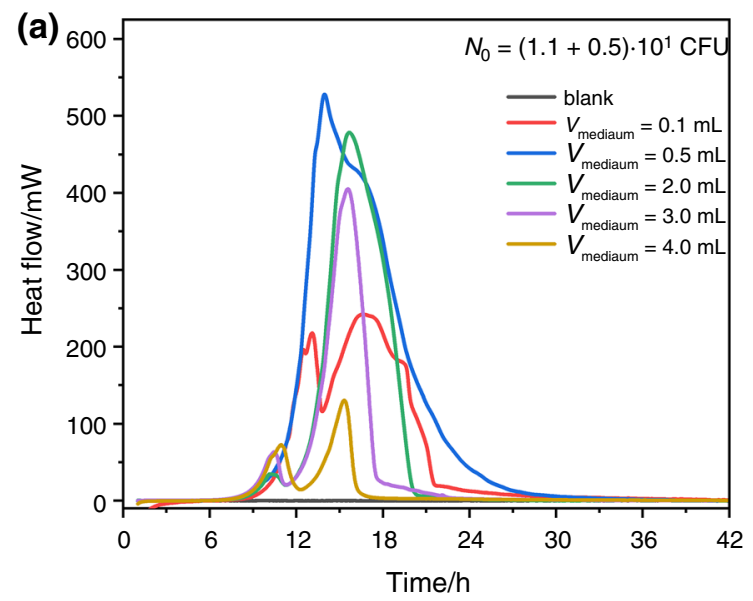

Fig. 10 Influence of $V_{\text {medium }}$ on the heat flow signals obtained from IMC experiments conducted in liquid medium. a Heat flow traces depending on $V_{\text {medium }}$. b Relationship between the detection time and
$V_{\text {medium }}$ (see Fig. 10a). However, the maximum of the second peak was determined by oxygen transferred from the headspace. Another exception was found at a threshold value of $50 \mu \mathrm{W}$ (blue points, Fig. 10b). For low $V_{\text {medium }}$, the detection time followed the linear trend from the previous observation. However, the detection time decreased at a $V_{\text {medium }}$ of $3 \mathrm{~mL}$ and dropped to a value of approx. $10.5 \mathrm{~h}$ at a $V_{\text {medium }}$ of $4.0 \mathrm{~mL}$ (Fig. 10b). When using 3 or $4 \mathrm{~mL}$ instead of $2 \mathrm{~mL}$ filling volume, the dissolved amount of oxygen was so large that the initial peak reached a detectable heat flow of more than $50 \mu \mathrm{W}$. The large error bars in the case of the measurement point at $3 \mathrm{~mL}$ (Fig. 10b) are since only in one of three measurements a heat flow of the initial peak exceeded $50 \mu \mathrm{W}$ and was therefore detectable. The detection of the other two curves only occurred at the second peak (temporally delayed, see explanation above) which then exceeded $50 \mu \mathrm{W}$. A summary of all $V_{\text {medium }}$-dependent heat flow signals measured on SC and LC is given in chapter S7 in the SI. As our data showed, for aerobic bacteria to be detected in LC it is important to know how much dissolved oxygen is needed to produce a detectable heat flow. This value can be estimated by using the following eq:

$\Phi_{\text {dect }}<\mu \cdot c_{\mathrm{O}_{2}} \cdot \Delta_{k} H_{\mathrm{O}_{2}}+N_{0} \cdot \varphi_{0}$

where $c_{\mathrm{O}_{2}}$ and $\Delta_{\mathrm{k}} H_{\mathrm{O}_{2}}$ are the amount of dissolved oxygen in the aqueous medium and the oxycaloric equivalent $\left(-455 \mathrm{~kJ} \mathrm{~mol}^{-1} \mathrm{O}_{2}\right)$ [32], respectively. The derivation of Eq. (6) is given in chapter S8 of the SI.

\section{Influencing biological heat production}

Cell-specific heat production rate $\varphi_{0}$ and specific growth rate $\mu$ are characteristic of the respective bacterial strain.

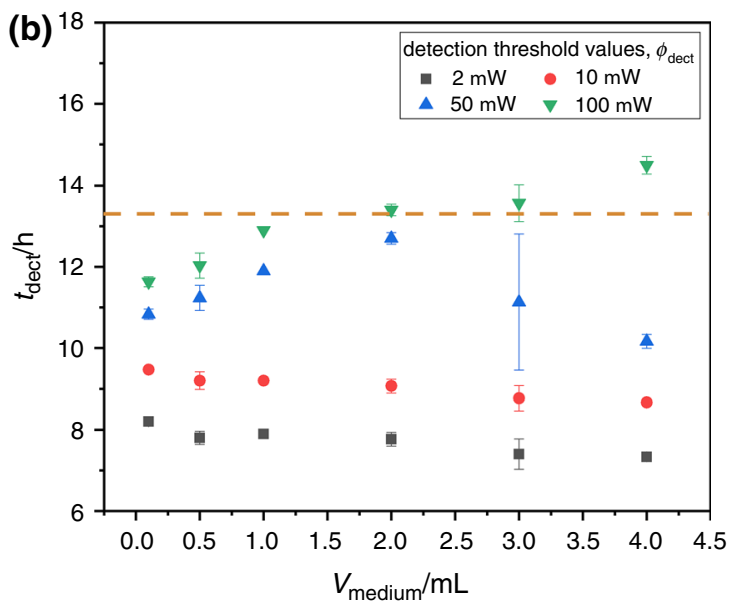

$V_{\text {medium }}$ as a function of different detection threshold values $\Phi_{\text {dect }}=2$, 10,50 and $100 \mu \mathrm{W}$. For comparison, the dashed line in $\mathbf{b}$ indicates the mean CFU detection time determined by visual inspection 
High values of both favour early calorimetric detection. The growth rate likewise favours conventional visual detection and is affected by environmental conditions like temperature, $\mathrm{pH}$, salinity or substrate availability [45-47]. All of these factors can be adjusted to achieve optimal growth. It is worth mentioning here that IMC can be advantageous for screening of optimal growth conditions [48], as all the factors mentioned influence the metabolism of the bacterial cell which is strongly correlated with the heat released [49].

A simple method to achieve fast growth is the use of complex media (e.g. lysogeny broth LB or trypticase soy broth TSB) [50]. These media contain important building blocks and provide all necessary components such as amino acids, carbohydrates and vitamins etc. [51] for the biosynthesis (anabolism) [52]. Thus, the build-up of bacterial biomass can take place faster and more energy efficiently than by synthesis from one or a small set of substrate molecules. To demonstrate this difference in terms of detection, we performed another IMC experiment in LC by using a minimal medium (MMKT2440) instead of DMS-1 medium. Everything else remained the same as described in Fig. 2b. For comparison, Fig. 11 shows the heat flow signals of bacterial growth in minimal medium as well as in DMS-1 medium (taken from Fig. 6a). For details, the corresponding heat flow signals measured in MMKT2440 are shown in chapter S9 in the SI.

A comparison of the heat flow signals showed that bacterial growth can be easily stimulated by a selective choice of the medium composition. Consequently, it is beneficial for the practitioner if metabolic processes of the desired target microorganisms are known and thus the detection time might be reduced via the medium composition. Complementary to this, it should be emphasized that this consideration also applies to temperature and $\mathrm{pH}$ as demonstrated by KATARAO et al. using the example of E. coli [49].

The cell-specific heat production rate is poorly investigated since metabolic processes of bacteria involve heat flows in the picowatt range. The mean cell-specific heat production rate is used, but this was only determined for a few bacteria under different growth conditions (e.g. aerobic, anaerobic, exponentially growing, resting) [4, 53-55]. Any possibility to increase the cellular heat with, e.g. selective substrates or reagents would favour an IMC detection of bacterial contaminations as they would allow earlier, more sensitive and more selective detection. The kind of metabolism might also play a key role since respiratory growth often releases more heat than fermentative growth [42]. One would thus cultivate facultative anaerobes under oxic conditions to increase the mean cell-specific heat production rate. For instance, the cell-specific heat production rate for $E$. coli is $0.8 \mathrm{pW} /$ cell during aerobic growth as opposed to $0.2 \mathrm{pW} /$ cell during anaerobic growth [17].

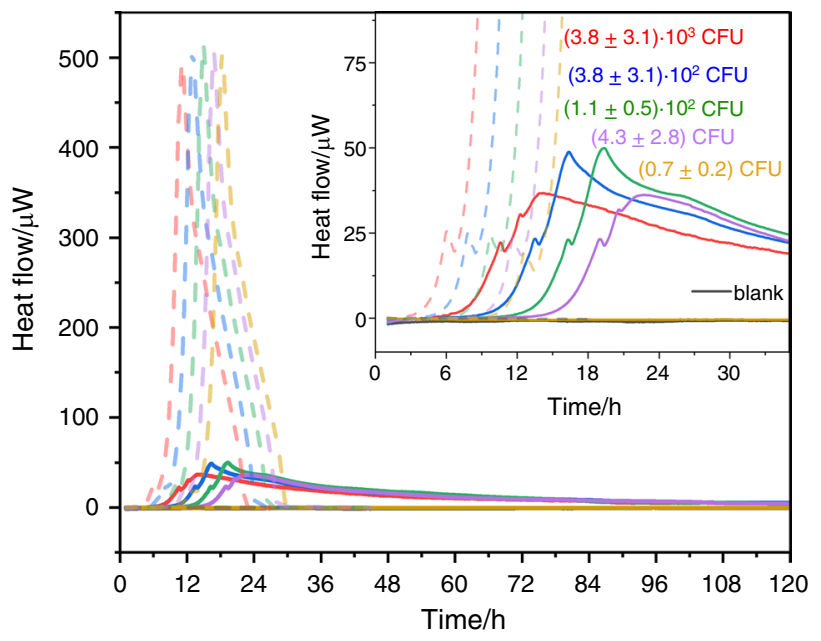

Fig. 11 Influence of the medium composition on the heat flow signals obtained by using complex medium (DSM-1, dashed lines) and chemical defined minimal medium (MMKT2440, solid lines). The insert provides a magnification of the heat flow signals. The $N_{0}$ (mean $\pm \mathrm{SD}$ ) in CFU are indicated next to the heat flow signals and based on the inoculum volume $(10 \mu \mathrm{L})$

\section{Conclusions}

Our study analysed the three factors determining the detection time in microcalorimetric measurements of aerobic bacterial contaminations in 4-mL static ampoules: (1) the initial number of bacteria $\left(N_{0}\right),(2)$ the performance of the applied isothermal microcalorimeter (IMC) and (3) the provision of substrate and oxygen in the solid- or liquid-based cultivation set-up. This study used the aerobically growing $P$. putida mt-2 KT2440 as an example and complements an analogous study conducted with an anaerobic system [25]. This is important because numerous pathogenic or other bacterial contaminants grow aerobically.

The current trend in the field of IMC detection of bacterial contaminations is that, on the one hand, less powerful calorimeters (applicable detection thresholds $>10 \mu \mathrm{W}$ ) use large sample vessels (up to $125 \mathrm{~mL}$ sample volume) but have only a few measuring channels. On the other hand, powerful calorimeters (applicable detection thresholds $<10 \mu \mathrm{W}$ ) are used which have small sample vessels and allow up to 48 simultaneous measurements. Our results, together with the applications already known from the literature, e.g. $E$. coli produced a max. heat flow of $100 \mu \mathrm{W}\left(33 \mathrm{~mW} \mathrm{~L}^{-1}\right)$ in the concentration range of $10^{5}$ to $10^{0} \mathrm{CFU} \cdot \mathrm{mL}^{-1}$ [56] or approx. $650 \mu \mathrm{W}\left(217 \mathrm{~mW} \mathrm{~L}^{-1}\right)$ in the concentration range of $10^{5}$ to $10^{0} \mathrm{CFU} \mathrm{mL} \mathrm{m}^{-1}$ [19] (depending on the cultivation conditions, for details see the respective reference), Staphylococcus aureus produced a max. heat flow of 100 to $350 \mu \mathrm{W}$ (33 to $117 \mathrm{~mW} \mathrm{~L}^{-1}$ ) depending on the concentration ( $10^{5}$ to $\left.10^{0} \mathrm{CFU} \mathrm{mL}^{-1}\right)$ [19] and Legionella pneumophila produced a max. heat flow of $175 \mu \mathrm{W}\left(58 \mathrm{~mW} \mathrm{~L}^{-1}\right)$ in the 
concentration range of $10^{5}$ to $10^{2} \mathrm{CFU} \mathrm{mL}^{-1}$ [57], show that it might be possible to develop a simple calorimeter that is less powerful (high detection threshold), uses small sample vessels $(4-5 \mathrm{~mL})$ and has a sufficient number of channels. The detection of metabolically induced heat flows in the range of $50-100 \mu \mathrm{W}$ and the use of $4-\mathrm{mL}$ ampoules might be sufficient for most applications. Due to the exponential growth behaviour of the bacteria, the detection delay is only a few hours compared to powerful but also very expensive IMCs. We were also able to show that an increase in the initial number of bacteria can be a feasible solution. As an extension, especially for low contaminations or if larger sample volumes are investigated which would not be appropriate for 4-mL ampoules, membrane filtration would be a reasonable option. Additionally, if membranes with selectively antimicrobial surfaces or pore sizes [58] would be used, calorimetric detection could even be combined with the reduction of the influence of a potential accompanying bacterial flora. This should be the subject of follow-up research because bacterial contaminations are sometimes caused by bacterial communities and not only by single strains. However, there are already some potentially alternative enrichment methods such as biomagnetic separation (BMS) which has been successfully applied in the rapid detection of E. coli [59].

We were able to show that even less powerful calorimeters which only recognize a detectable heat flow of $50 \mu \mathrm{W}$ and more, can show comparatively early detection despite oxygen diffusion problems in the case of liquid cultivation (LC). However, very low $N_{0}$ on solid cultivation (SC) were critical, since our experiments have shown that if only a few colonies grow on the solid medium, a transition between exponential and linear heat flow can be observed. This behaviour is due to substrate diffusion-limited linear growth of colonies. Cultivation on solid substrate can be considered as a useful alternative, especially for microorganisms that grow better or only on solid medium. In the case of strictly aerobic growing bacteria, $\mathrm{SC}$ offers better access to oxygen [23]. Furthermore, lager $V_{\mathrm{i}}$ might be also applicable in SC by increasing the cross-sectional area of calorimetric vessels.

Finally, it should be noted that a deeper understanding in biological characteristics like stimulating specific growth rate $\mu$ through medium composition, temperature or $\mathrm{pH}$ as well as the cell-specific heat production rate $\varphi_{0}$ which is still comparatively little investigated, bears an enormous potential to further reduce detection times. Concerning $\varphi_{0}$, one possibility could be the action of targeted substrates to stimulate the metabolic response of the microorganisms to yield a greater cell-specific heat production rate.

Acknowledgements Open Access funding provided by Projekt DEAL. The authors acknowledge M. Kolbe (UFZ, Department of Environmental Microbiology) and K. Lübke (UFZ, Department of Environmental
Microbiology) for preparing the media and N. Ghanem, T. Mainka, S. Paufler, C. Heber, A. Krämer and M. Reichard (UFZ Department of Environmental Microbiology) for technical support.

Funding This work was funded by the German Federation of Industrial Research Associations (AiF BMWi, AiF-Nr. ZF4315807RH7).

\section{Compliance with ethical standards}

Conflict of interest The authors declare that the research was conducted in the absence of any commercial or financial relationships that could be construed as a potential conflict of interest.

Open Access This article is licensed under a Creative Commons Attribution 4.0 International License, which permits use, sharing, adaptation, distribution and reproduction in any medium or format, as long as you give appropriate credit to the original author(s) and the source, provide a link to the Creative Commons licence, and indicate if changes were made. The images or other third party material in this article are included in the article's Creative Commons licence, unless indicated otherwise in a credit line to the material. If material is not included in the article's Creative Commons licence and your intended use is not permitted by statutory regulation or exceeds the permitted use, you will need to obtain permission directly from the copyright holder. To view a copy of this licence, visit http://creativecommons.org/licenses/by/4.0/.

\section{References}

1. Wadsö I, Goldberg RN. Standards in isothermal microcalorimetry (IUPAC Technical Report). Pure Appl Chem. 2001;73:1625-39.

2. Gustafsson L. Microbiological calorimetry. Thermochim Acta. 1991;193:145-71.

3. Ladbury JE, Chowdhry BZ. Sensing the heat: the application of isothermal titration calorimetry to thermodynamic studies of biomolecular interactions. Chem Biol. 1996;3:791-801.

4. Kemp RB. An historical review of developments in cellular microcalorimetry. Pure Appl Chem. 1993;65:1875-80.

5. Wadsö L, Gómez GF. Isothermal calorimetry for biological applications in food science and technology. Food Control. 2009;20(10):956-61.

6. Bruylants G, Wouters J, Michaux C. Differential scanning calorimetry in life science: thermodynamics, stability, molecular recognition and application in drug design. Curr Med Chem. 2005;12:2011-20.

7. Ozawa T. Thermal analysis-review and prospect. Thermochim Acta. 2000;355:35-42.

8. Wadsö I. Microcalorimetric techniques for characterization of living cellular systems. will there be any important practical applications? Thermochim Acta. 1995;269-270:337-50.

9. Maskow T, Kemp R, Buchholz F, Schubert T, Kiesel B, Harms H. What heat is telling us about microbial conversions in nature and technology: from chip- to megacalorimetry. Microbiol Biotechnol. 2010;3:269-84.

10. Lorinczy D. Thermal analysis in biological and medical applications. J Therm Anal Calorim. 2017;130:1263-80.

11. Braissant O, Wirz D, Göpfert B, Daniels AU. Biomedical use of isothermal microcalorimeters. Sensors. 2010;10:9369-83.

12. Lamprecht I. Calorimetry and thermodynamics of living systems. Thermochim Acta. 2003;405:1-13.

13. Stenesh J. Introduction to metabolism biochemistry. Boston: Springer; 1998. p. 203-19. 
14. von Stockar U, Liu JS. Does microbial life always feed on negative entropy? Thermodynamic analysis of microbial growth. Biochim Biophys Acta Bioenerg. 1999;1412:191-211.

15. Maskow T, Wolf K, Kunze W, Enders S, Harms H. Rapid analysis of bacterial contamination of tap water using isothermal calorimetry. Thermochim Acta. 2012;543:273-80.

16. Brueckner D, Krähenbühl S, Zuber U, Bonkat G, Braissant O. An alternative sterility assessment for parenteral drug products using isothermal microcalorimetry. J Appl Microbiol. 2017;123:773-9.

17. Maskow T, Schubert T, Wolf A, Buchholz F, Regestein L, Buechs J, et al. Potentials and limitations of miniaturized calorimeters for bioprocess monitoring. Appl Microbiol and Biotechnol. 2011;92:55.

18. Chen J, Li K, Liu C, Li M, Lv Y, Jia L, et al. Enhanced efficiency of thermoelectric generator by optimizing mechanical and electrical structures. Energies. 2017;10:1329.

19. Trampuz A, Salzmann S, Antheaume J, Daniels AU. Microcalorimetry: a novel method for detection of microbial contamination in platelet products. Transfusion. 2007;47:1643-50.

20. Boling EA, Blanchard GC, Russell WJ. Bacterial identification by microcalorimetry. Nature. 1973;241:472-3.

21. Chang-Li X, Hou-Kuhan T, Zhau-Hua S, Song-Sheng Q, Yao-Ting L, Hai-Shui L. Microcalorimetric study of bacterial growth. Thermochim Acta. 1988;123:33-41.

22. Bonkat G, Braissant O, Rieken M, Solokhina A, Widmer AF, Frei R, et al. Standardization of isothermal microcalorimetry in urinary tract infection detection by using artificial urine. World J Urol. 2013;31:553-7.

23. Braissant O, Wirz D, Gopfert B, Daniels AU. "The heat is on": rapid microcalorimetric detection of mycobacteria in culture. Tuberculosis. 2010;90:57-9.

24. Koga K, Nishizawa YU, Matsumoto Y-I, Hara T, Takahashi $\mathrm{K}$. Evaluation of the growth activity of escherichia coli and staphylococcus aureus colonies on solid medium using microbial calorimetry. Biocontrol Sci. 2004;9:21-8.

25. Fricke C, Harms H, Maskow T. Rapid calorimetric detection of bacterial contamination: influence of the cultivation technique. Front Microbiol. 2019;10:1-12.

26. Monod J. The growth of bacterial cultures. Annu Rev Microbiol. 1949;3:371-94

27. Saha K. The Earth's atmosphere: its physics and dynamics. Berlin: Springer; 2008

28. Benson BB, Krause D Jr. The concentration and isotopic fractionation of oxygen dissolved in freshwater and seawater in equilibrium with the atmosphere. Limnol Oceanogr. 1984;29:620-32.

29. Hazan R, Que Y-A, Maura D, Rahme LG. A method for high throughput determination of viable bacteria cell counts in 96-well plates. BMC Microbiol. 2012;12:1-7.

30. Hoffmann S, Walter S, Blume A-K, Fuchs S, Schmidt C, Scholz A, et al. High-throughput quantification of bacterial-cell interactions using virtual colony counts. Front Cell Infect Microbiol. 2018;8:1-10.

31. Bonkat G, Bachmann A, Solokhina A, Widmer AF, Frei R, Gasser $\mathrm{TC}$, et al. Growth of mycobacteria in urine determined by isothermal microcalorimetry: implications for urogenital tuberculosis and other mycobacterial infections. Urology. 2012;80:1163.e9-. e12.

32. Gnaiger E, Kemp RB. Anaerobic metabolism in aerobic mammalian cells: information from the ratio of calorimetric heat flux and respirometric oxygen flux. Biochim Biophys Acta Bioenerg. 1990;1016:328-32.

33. Bartram J, Cotruvo JA, Exner M, Fricker C, Glasmacher A. Heterotrophic plate counts and drinking-water safety. Geneva: World Health Organization; 2003.
34. Braissant O, Wirz D, Göpfert B, Daniels AU. Use of isothermal microcalorimetry to monitor microbial activities. FEMS Microbiol Lett. 2010;303:1-8.

35. Bäckman P, Bastos M, Hallén D, Lönnbro P, Wadsö I. Heat conduction calorimeters: time constants, sensitivity and fast titration experiments. J Biochem Biophys Methods. 1994;28:85-100.

36. Wadsö I. Isothermal microcalorimetry near ambient temperature: an overview and discussion. Thermochim Acta. 1997;294:1-11.

37. Lee W, Fon W, Axelrod BW, Roukes ML. High-sensitivity microfluidic calorimeters for biological and chemical applications. Proc Natl Acad Sci USA. 2009;106:15225-30.

38. Rodríguez D, Daniels AU, Urrusti JL, Wirz D, Braissant O. Evaluation of a low-cost calorimetric approach for rapid detection of tuberculosis and other mycobacteria in culture. J Appl Microbiol. 2011;111:1016-24.

39. Altwasser V, Patz RR, Lemke T, Paufler S, Maskow T. A simple method for the measurement of metabolic heat production rates during solid-state fermentations using ss-carotene production with Blakeslea trispora as a model system. Eng Life Sci. 2017;17:620-8.

40. Braissant O, Theron G, Barnard M, Friedrich SO, Diacon AH, Bonkat G. Comparison of isothermal microcalorimetry and BACTEC MGIT960 for detection of the metabolic activity of Mycobacterium tuberculosis in sputum samples. J Appl Microbiol. 2019;128:1497-502.

41. Crabbé A, Leroy B, Wattiez R, Aertsen A, Leys N, Cornelis P, et al. Differential proteomics and physiology of Pseudomonas putida KT2440 under filament-inducing conditions. BMC Microbiol. 2012;12:1-9.

42. Maskow T, Morais FM, Rosa LFM, Qian YG, Harnisch F. Insufficient oxygen diffusion leads to distortions of microbial growth parameters assessed by isothermal microcalorimetry. RSC Adv. 2014;4:32730-7.

43. Tronnolone H, Tam A, Szenczi Z, Green JEF, Balasuriya S, Tek EL, et al. Diffusion-limited growth of microbial colonies. Sci Rep. 2018;8:1-11.

44. Warren M, Hwa T. The growth of bacterial colonies. Biophys J. 2012;102:152a.

45. Stoward PJ. Thermodynamics of biological growth. Nature. 1962;194:977-8.

46. Egli T. Microbial growth and physiology: a call for better craftsmanship. Front Microbiol. 2015;6:1-12.

47. Antoniou P, Hamilton J, Koopman B, Jain R, Holloway B, Lyberatos $\mathrm{G}$, et al. Effect of temperature and ph on the effective maximum specific growth rate of nitrifying bacteria. Water Res. 1990;24:97-101.

48. Zaharia DC, Muntean AA, Popa MG, Steriade AT, Balint O, Micut R, et al. Comparative analysis of Staphylococcus aureus and Escherichia coli microcalorimetric growth. BMC Microbiol. 2013;13:1-14.

49. Katarao A, Yamato N, Takahashi K. Calorimetric study of Escherichia coli growth on Bouillon medium. Agric Biol Chem. 1987;51:2437-42.

50. Sezonov G, Joseleau-Petit D, D’Ari R. Escherichia coli physiology in Luria-Bertani broth. J Bacteriol. 2007;189:8746-9.

51. Burrows W. The nutritional requirements of bacteria. Q Rev Biol. 1936;11:406-24.

52. Russell JB, Cook GM. Energetics of bacterial growth: balance of anabolic and catabolic reactions. Microbiol Rev. 1995;59:48-62.

53. Loesberg C, van Miltenburg JC, van Wuk R. Heat production of mammalian cells at different cell-cycle phases. J Therm Biol. 1982;7:209-13.

54. Kimura T, Takahashi K. Calorimetric studies of soil microbes: quantitative relation between heat evolution during microbial 
degradation of glucose and changes in microbial activity in soil. Microbiology. 1985;131:3083-9.

55. Robador A, LaRowe DE, Finkel SE, Amend JP, Nealson KH. Changes in microbial energy metabolism measured by nanocalorimetry during growth phase transitions. Front Microbiol. 2018;9:1-7.

56. Bonkat G, Braissant O, Widmer AF, Frei R, Rieken M, Wyler $\mathrm{S}$, et al. Rapid detection of urinary tract pathogens using microcalorimetry: principle, technique and first results. BJU Int. 2012;110:892-7.

57. Fricke C, Xu J, Jiang FL, Liu Y, Harms H, Maskow T. Rapid culture-based detection of Legionella pneumophila using isothermal microcalorimetry with an improved evaluation method. Microbiol Biotechnol. 2020;13:1262-72.
58. Bobbitt JA, Betts RP. The removal of bacteria from solutions by membrane filtration. J Microbiol Methods. 1992;16:215-20.

59. Lerchner J, Schulz A, Poeschel T, Wolf A, Hartmann T, Mertens F, et al. Chip calorimetry and biomagnetic separation: fast detection of bacterial contamination at low cell titers. Eng Life Sci. 2012;12:615-20.

Publisher's Note Springer Nature remains neutral with regard to jurisdictional claims in published maps and institutional affiliations. 